\title{
Spin dependence of ferroelectric polarization in the double exchange model for manganites
}

\author{
I. V. Solovyev ${ }^{1,2, *}$ and S. A. Nikolaev ${ }^{2}$ \\ ${ }^{1}$ Computational Materials Science Unit, \\ National Institute for Materials Science, \\ 1-1 Namiki, Tsukuba, Ibaraki 305-0044, Japan \\ ${ }^{2}$ Department of Theoretical Physics and Applied Mathematics, \\ Ural Federal University, Mira str. 19, 620002 Ekaterinburg, Russia
}

(Dated: August 13, 2018) 


\begin{abstract}
The double exchange (DE) model is systematically applied for studying the coupling between ferroelectric (FE) and magnetic orders in several prototypical types of multiferroic manganites. The model itself was constructed for the magnetically active Mn $3 d$ bands in the basis of Wannier functions and include the effect of screened on-site Coulomb interactions in the Hartree-Fock approximation. All model parameters were derived from the first-principles electronic structure calculations. The essence of our approach for the FE polarization is to use the Berry phase theory, formulated in terms of occupied Wannier functions, and to evaluate the asymmetric spin-dependent change of these functions in the framework of the DE model. This enables us to quantify the effect of the magnetic symmetry breaking and derive several useful expressions for the electronic polarization $\mathbf{P}$, depending on the relative directions of spins. The spin-dependence of $\mathbf{P}$ in the DE model is given by the isotropic correlation functions $\mathbf{e}_{i} \cdot \mathbf{e}_{j}$ between directions of neighboring spins. Despite formal similarity with the magnetostriction mechanism, the magnetoelectric (ME) coupling in the proposed DE theory is not related to the magnetically driven FE atomic displacements and can exist even in compounds with the centrosymmetric crystal structure, if the spacial distribution of $\mathbf{e}_{i} \cdot \mathbf{e}_{j}$ does not respect the inversion symmetry. The proposed theory is applied to the solution of three major problems: (i) The magnetic-state dependence of $\mathbf{P}$ in hexagonal manganites, using $\mathrm{YMnO}_{3}$ as an example; (ii) The microscopic relationship between canted ferromagnetism and $\mathbf{P}$ in monoclinic $\mathrm{BiMnO}_{3}$; (iii) The origin of $\mathrm{FE}$ activity in orthorhombic manganites. Particularly, we will show that for an arbitrary noncollinear magnetic structure, propagating along the orthorhombic $\boldsymbol{b}$ axis and antiferromagnetically coupled along the $\boldsymbol{c}$ axis, $\mathbf{P}$ is induced by an inhomogeneous distribution of spins and can be obtained by scaling the one of the E-type antiferromagnetic (AFM) phase with the prefactor depending only on the relative directions of spins and being the measure of this spin inhomogeneity. This picture works equally well for the twofold $\left(\mathrm{HoMnO}_{3}\right)$ and fourfold $\left(\mathrm{TbMnO}_{3}\right)$ periodic manganites. The basic difference is that, even despite some spin canting of the relativistic origin and deviation from the collinear E-type AFM aligment, the twofold periodic magnetic structure remains strongly inhomogeneous, that leads to large $\mathbf{P}$. On the contrary, the fourfold periodic magnetic structure can be viewed as a moderately distorted homogeneous spin spiral, which corresponds to much weaker $\mathbf{P}$.
\end{abstract}




\section{INTRODUCTION}

The multiferroic materials, which simultaneously exhibit a long-range magnetic order and a spontaneous electric polarization, have attracted a great deal of attention due to their potential applications in the next-generation electronic devises as well as the fundamental interest in the origin of magnetoelectric (ME) coupling. $\frac{1}{*}$ Among them, there is a very important subclass of materials, which are called "improper multiferroics", where the magnetic order does not simply coexist with the spontaneous polarization, but breaks the inversion symmetry by itself and, thus, becomes primarily responsible for the ferroelectric (FE) activity. Because of this intrinsic interconnection between polarization and magnetism, such materials are expected to exhibit a strong ME coupling.

There is a large number of theoretical studies, which introduce and emphasize the importance of different mechanisms of the ME coupling, associated with the spin current; $\stackrel{2}{=}$ nonrelativistic magnetostriction; $\underline{3}^{-\underline{6}}$ inverse Dzyalishinskii-Moriya (DM) mechanism, which is another type of magnetostriction, caused by the relativistic spin-orbit (SO) coupling: $;^{7}$ and spin-dependent $p$ - $d$ hybridization. ${ }^{-}$Currently, most of these theories have a phenomenological status, as each of them is typically oriented on the description of properties, observed in some narrow group of materials. Presumably, the most striking example is the properties of orthorhombic manganites, which are typically interpreted from two completely different standpoints: the FE activity in the twofold periodic systems ( such as $\mathrm{HoMnO}_{3}$ and $\mathrm{YMnO}_{3}$ ) is ascribed solely to the nonrelativistic magnetostriction mechanism, whereas in materials with longer magnetic periodicity ( such as $\mathrm{TbMnO}_{3}$ ) it is believed to have a purely relativistic origin, associated with either the spin current or the inverse DM mechanism.

In this work we continue to develop the double exchange (DE) theory of the ME coupling, $\underline{9}$ which is oriented on the wide class of multiferroic manganites with different types of the crystallographic and magnetic structure.

Manganites play one of the key roles in the materials science engineering of novel multiferroic compounds. There are two reasons for it: (i) The orbital ordering, which, in the combination with other factors, assists the antisymmetric charge transfer and the formation of the spontaneous polarization; $; 10,11$ (ii) The high spin state of the $\mathrm{Mn}^{3+}$ ions, which is driven by intraatomic Hund's coupling and plays a crucial role in the magnetic inversion symmetry breaking: since the nonmagnetic state of the $\mathrm{Mn}^{3+}$ sites would lead to a gigantic loss 
of the intraatomic Hund's energy, in certain magnetic structures with competing magnetic interactions, it is more favorable energetically to keep these sites magnetic, but to abandon the inversion symmetry. 12

The basic electronic and magnetic properties of manganites are described by the DE model. $\underline{13}, \underline{14}$ Although the concept of the double exchange was originally introduced for the analysis of the metallic state, realized in hole-doped manganites, today it is understood much more generally - as a generic property of high spin compounds with the partially filled majority-spin states. From a mathematical point of view, the 'high spin state' means that the intraatomic exchange splitting between the majority- and minority-spin states is so large that the effect of the latter states on the considered properties can be neglected.

The reorientation of spins in the DE model may lead to a dramatic change of the electronic structure and even open the band gap $\stackrel{14-16}{\underline{16}}$ Therefore, in order to understand the behavior of the FE polarization in the DE model, it is very important to link it to the change of the electronic structure. This can be achieved by using the Berry phase theory of polarization, which can be reformulated in terms of the occupied Wannier functions in the real space.17,18 For compounds with the centrosymmetric crystal structure, such procedure naturally gives us the electronic polarization, induced by a noncentrosymmetric magnetic order.

Hopefully, the electronic structure of insulating manganites is characterized by another large parameter $\Delta$, which is the splitting between the occupied and unoccupied states with the same spin. It is caused by the Jahn-Teller distortion and additionally enhanced by the screened on-site Coulomb repulsion. This enables us to use the perturbation theory in order to evaluate the asymmetric spin-dependent change of the Wannier functions in the first order of $1 / \Delta . \underline{9}$ This change will automatically gives the spin dependence of the electronic polarization.

Needless to say that this mechanism of the ME coupling is essentially nonrelativistic one: The relativistic spin-orbit interaction can play an important role in stabilizing noncentrosymmetric magnetic structures. However, once it is known, the FE polarization can be described by the nonrelativistic DE theory. Therefore, depending on the type of the magnetic structure, this mechanism can produce a large FE polarization.

In any model analysis, in order to describe the properties of realistic materials and to elucidate the differences between these materials, it is very important to make a link to the first-principles calculations. In our case all parameters of the DE model are derived 
from the first-principles electronic structure calculations, by constructing some effective Hamiltonians for the magnetically active states in the basis of Wannier functions. ${ }^{19}$ Such a procedure typically gives us very reliable description of multiferroic and other properties of transition-metal oxides at a semi-quantitative level. ${ }^{20}-23$

In our previous work (Ref. 9), we have applied such DE model for the analysis of ferroelectric (FE) activity in one particular type of manganites, crystallizing in the orthorhombic structure and forming twofold periodic magnetic structure in the ground state. We have argued that by using the DE model one can indeed successfully rationalize many aspects of the FE activity in this type of systems. Moreover, even at a quantitative level, it reproduces results of more general mean-field Hartree-Fock calculations for the effective model, which were used as the starting point for the construction of the DE model. These results are also in a good agreement with available first-principles electronic structure calculations. $., 24,25$

In this work we will systematically apply the DE model for the analysis of the wide class of multiferroic manganites. We will show that the double exchange is indeed the key microscopic mechanism, which explains the basic aspects of the FE activity, related to the interplay between magnetic and crystallographic symmetries in various types of manganites.

The rest of the article is organized as follows. In Sec. II, we will introduce the DE model of the ME coupling in multiferroic manganites. In Sec. III, we will discuss applications of this model for different types of manganites. Particularly, we will consider the orthorhombic systems (the space group Pbnm), forming twofold and fourfold periodic magnetic structures in the ground state (Sec. IIIA), the monoclinic $C 2 / c$ phase of $\mathrm{BiMnO}_{3}$ (Sec. IIIB), and hexagonal manganites, crystallizing in the $P 6_{3} \mathrm{~cm}$ structure, using $\mathrm{YMnO}_{3}$ as an example (Sec. IIIC). Finally, in Sec. IV], we will present a summary of our work.

\section{DOUBLE EXCHANGE MODEL FOR MAGNETOELECTRIC COUPLING}

Our strategy consists of the following steps:

(i) We assume that FE and magnetic properties in the ground state of considered systems can be described reasonably well at the level of the mean-field theories. It can be the Kohn-Sham density functional theory or its refinements, ${ }^{26}$ which are widely used in the

first-principles electronic structure calculations. In our case, we focus on the behavior of magnetically active $3 d$ bands of manganites and replace the first-principles calculations for 
this part by the solution of the realistic Hubbard-type model, which was rigorously constructed using the technique of Wannier functions. $\underline{19}, \underline{27}, \underline{28}$ More specifically, we start with the electronic band structure in the local-density approximation (LDA), construct the Wannier functions for the $3 d$ bands, and calculate the matrix elements of the LDA Hamiltonian in the basis of these Wannier functions. Such a construction gives us the proper one-electron part of the model Hamiltonian. Then, we calculate the screened on-site Coulomb interactions for the $3 d$ bands, using the simplified version of the constrained random-phase approximation, $\underline{29}$ as explained in Ref. 19. After that, we solve the effective Hubbard-type model in the meanfield Hartree-Fock (HF) approximation. The solution gives us the mean-field Hamiltonian of the form:

$$
\hat{H}_{i j}^{\mathrm{MF}}=\hat{t}_{i j}+\hat{\mathcal{V}}_{i} \delta_{i j}
$$

Here, $\hat{t}_{i j}=\left[t_{i j}^{m m^{\prime}} \delta_{s s^{\prime}}\right]$ is the proper one-electron part of the Hubbard model between sites $i$ and $j$ in the basis of Wannier orbitals $m\left(m^{\prime}\right)=x y, y z, 3 z^{2}-r^{2}, z x$, and $x^{2}-y^{2}$, and $\hat{\mathcal{V}}_{i}$ is the self-consistent HF potential at the site $i$, which is constructed from the screened Coulomb interactions and the density matrix. $\underline{\underline{19}}$ The Wannier functions themselves were constructed using the projector-operator technique (Refs. 19 and 28) and the orthonormal linear muffin-tin orbitals (LMTO) (Ref. 30) as the trial wave functions. As the LMTO basis is already well localized, such procedure allows us to generate well localized Wannier functions. Therefore, the obtained transfer integrals $\left(t_{i j}^{m m^{\prime}}\right.$ for $\left.i \neq j\right)$ are typically restricted by the nearest neighbors, while other contributions are substantially smaller. Since the LDA band structure is nonmagnetic and we do not consider explicitly the SO coupling, the matrix $\hat{t}_{i j}$ does not depend on the spin indices ( $s$ and $s^{\prime}=\uparrow$ or $\downarrow$ ). Without SO interaction, $\hat{\mathcal{V}}_{i}$ is diagonal with respect to $s$ and $s^{\prime}$ :

$$
\hat{\mathcal{V}}_{i}=\left(\begin{array}{cc}
\hat{\mathcal{V}}_{i}^{\uparrow} & 0 \\
0 & \hat{\mathcal{V}}_{i}^{\downarrow}
\end{array}\right),
$$

where each $\hat{\mathcal{V}}_{i}^{\uparrow, \downarrow}$ is the $5 \times 5$ matrix in the orbital subspace.

(ii) We assume that, to a good approximation, $\hat{\mathcal{V}}_{i}^{\downarrow}$ can be replaced by $\hat{\mathcal{V}}_{i}^{\downarrow} \approx \hat{\mathcal{V}}_{i}^{\uparrow}+\Delta_{\text {ex }}$, where $\Delta_{\text {ex }}$ is the averaged exchange splitting between the majority- and minority-spin states. Due to Hund's interactions, the splitting $\Delta_{\mathrm{ex}}$ is large in manganites. Therefore, the details of $\hat{\mathcal{V}}_{i}^{\downarrow}$ in the unoccupied part of the spectrum becomes relatively unimportant on the energy scale of $\Delta_{\mathrm{ex}}$. Moreover, one can consider the limit $\Delta_{\mathrm{ex}} \rightarrow \infty$, and replace $\hat{H}_{i j}^{\mathrm{MF}}$ by the DE 
Hamiltonian: 13,14

$$
\hat{H}_{i j}^{\mathrm{DE}}=\xi_{i j} \hat{t}_{i j}+\hat{\mathcal{V}}_{i}^{\uparrow} \delta_{i j}
$$

which operates in the subspace of $\uparrow$-spin states, in the local coordinate frame, specified by the directions of spins $\mathbf{e}_{i}=\left(\cos \varphi_{i} \sin \vartheta_{i}, \sin \varphi_{i} \sin \vartheta_{i}, \cos \vartheta_{i}\right)$. The prefactor $\xi_{i j}$ in Eq. (2) is given by the well known expression: ${ }^{14}$

$$
\xi_{i j}=\cos \frac{\vartheta_{i}}{2} \cos \frac{\vartheta_{j}}{2}+\sin \frac{\vartheta_{i}}{2} \sin \frac{\vartheta_{j}}{2} e^{-i\left(\varphi_{i}-\varphi_{j}\right)} .
$$

(iii) The next step is the calculation of the electronic polarization using the Berry-phase method. $\stackrel{17}{18}$ For our purpose, it is convenient to use the real-space formulation of this method, in terms of the occupied Wannier functions $w_{n}$. Then, the electronic polarization is given by:

$$
\mathbf{P}=-\frac{e}{V} \sum_{n=1}^{M} \int \mathbf{r} w_{n}^{2}(\mathbf{r}) d \mathbf{r},
$$

where $-e(e>0)$ is the electron charge, $V=L V_{0}$ is the volume of magnetic unit cell (with $V_{0}$ being the volume of the crystallographic cell and $L$ being the number of such cells), and the summation $n$ runs over the occupied bands. Alternatively, one can sum up unoccupied bands. This should give us $\mathbf{- P}$. It is important to note that we treat $\mathbf{P}$ as a nonrelativistic quantity, which does not explicitly depend on the SO coupling. Nevertheless, the latter can still define the directions $\mathbf{e}_{i}$ of spins in the ground state.

The spin dependence of $\mathbf{P}$ in Eq. (3) is accumulated in $w_{n}(\mathbf{r})$. Then, for each $n$, one can arbitrarily shift the origin of integration in Eq. (3). Since each $w_{n}(\mathbf{r})$ is normalized, the shifted integral and the original one will differ by some vector, which depends on the shift, but does not depend on the spin variables. Therefore, since we are interested only in the spin dependence of $\mathbf{P}$, we shift the origin of each integral in Eq. (3) to the position of that atomic site for which $w_{n}$ was constructed and drop all concomitant terms, which do not depend on the spin degrees of freedom.

(iv) Our next observation is that many manganites exhibit the Jahn-Teller distortion, which splits the occupied and unoccupied $e_{g}$ orbitals. This splitting is additionally enhanced by the on-site Coulomb repulsion. Thus, there is another large parameter $\Delta$, which characterizes the electronic structure of manganites. We will define it as the intraatomic energy splitting between the center of gravity of occupied manifold, consisting of three $t_{2 g}$ and one $e_{g}$ levels, and the unoccupied $e_{g}$ level (see Fig. 1). The large $\Delta$ allows us to use the 


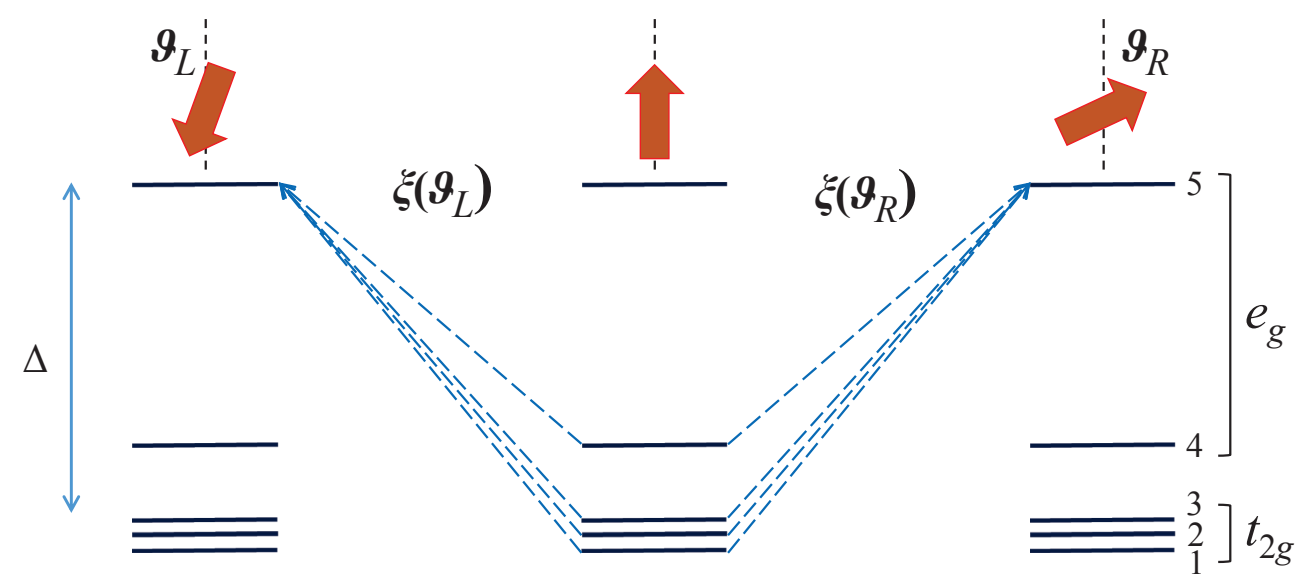

FIG. 1. (Color online) Schematic view, explaining the DE model for FE polarization in manganites: Due to specific magnetic alignment, the spins can form different angles in the bonds lying on the right $\left(\vartheta_{R}\right)$ and on the left $\left(\vartheta_{L}\right)$ relative to some center site. In the DE model, this leads to different scaling of the transfer integrals operating in these two bonds, which is described by the factors $\xi\left(\vartheta_{R}\right)$ and $\xi\left(\vartheta_{L}\right)$, respectively. Even if the central site is located in the inversion center, this magnetic alignment breaks the inversion symmetry. Using the Berry phase theory, 1,18 the polarization can be related to the spin-dependent asymmetric transfer of the weights of the occupied Wannier functions to the neighboring sites, which are given by transfer integrals between occupied and unoccupied orbitals (shown by dashed arrows). Since the unoccupied $e_{g}$ orbital splits off from the occupied ones by large crystal field and the Coulomb repulsion (the corresponding splitting is denoted by $\Delta$ ), these transfer integrals can be treated as a perturbation in the leading order of $1 / \Delta$.

perturbation theory for the occupied Wannier functions $w_{n}$ and evaluate their change in the first order of $1 / \Delta$. Practically, $\Delta$ is obtained form the diagonalization of the site-diagonal part $\left(\hat{t}_{i i}+\hat{\mathcal{V}}_{i}^{\uparrow}\right)$ of the DE Hamiltonian (2), which specifies the so-called crystal-field representation (if there are two types of $\mathrm{Mn}$ sites, like in $\mathrm{BiMnO}_{3}$, we use the averaged value of $\Delta$ ). Note that the occupied states in manganites are typically not well separated from each other and form one broad band. From this point of view, when we consider the energy splitting, it is more reasonable to use only one energy for all occupied states and take it in the center of gravity of these states.

The transfer integrals can be also transformed to the crystal-field representation: $\hat{t}_{i j} \rightarrow \hat{\mathrm{t}}_{i j}$. 
Then, one can start with the atomic limit, where all $w_{n}$ are fully localized on their atomic sites, and consider the transfer of weight of $w_{n}$ to the neighboring sites in the first order of $\hat{\mathrm{t}}_{i j} / \Delta$. Since the transfers within occupied states correspond to some unitary transformation of $w_{n}$, they will not change the physical properties. Thus, it is sufficient to consider only the transfer integrals, connecting the occupied and unoccupied orbitals in the crystal-field representation. As was pointed out above, alternatively, one can consider the change of the unoccupied states and the transfer integrals from the unoccupied to occupied orbitals, which is more convenient for our purposes. Since, at each Mn site, there is only one unoccupied orbital, one can drop the index $n$ in the notations of $w_{n}$ and replace it by the site index $i$. Furthermore, we adopt the lattice model and assume that all weights of $w$ are localized in the lattice points: i.e., if $w_{i}$ is centered at the site $i$, its weight can be presented in the form

$$
w_{i}^{2}(\mathbf{r})=\sum_{j} w_{i j}^{2} \delta\left(\mathbf{r}-\Delta \boldsymbol{\tau}_{j i}\right),
$$

where $\Delta \boldsymbol{\tau}_{j i}=\boldsymbol{R}_{j}-\boldsymbol{R}_{i}$ is the position of the site $j$ relative to the site $i$. Then, in the atomic limit, the weight is accumulated at the central site $\left(w_{i j}^{2}=1\right.$ and 0 for $j=i$ and $j \neq i$, respectively). Then, the weights at the neighboring sites can be obtained in the first order of the perturbation theory for $w_{i}$ as

$$
w_{i j}^{2}=\frac{1}{\Delta^{2}} \sum_{m \leq 4}\left|\mathrm{t}_{i j}^{5 m}\right|^{2},
$$

where the summation runs over four occupied orbitals. Since $\hat{t}_{j i}=\hat{t}_{i j}^{T}$, the weights $w_{i j}^{2}$ and $w_{j i}^{2}$ can be obtained from the same matrix of transfer integrals.

(v) Finally, in the DE model, the transfer integrals $\hat{t}_{i j}$ should be additionally modulated by $\xi_{i j}$, which depends on the relative orientation of spins at the sites $i$ and $j$. Thus, even though the crystal structure itself is centrosymmetric and the Mn sites are located in the inversion centers (like in orthorhombic manganites, crystallizing in the Pbnm structure), the multipliers $\xi_{i j}$ can make some bonds, connecting the central Mn site with its neighbors, inequivalent. For example, if the right bond $0 R$ in Fig. 1 is transformed to the left bond $0 L$ by the inversion operation, the spin alignment yielding $\left|\xi\left(\vartheta_{R}\right)\right| \neq\left|\xi\left(\vartheta_{L}\right)\right|$ will make these bonds inequivalent. This will break the inversion symmetry and produce some 'dipole', associated with the site $i$, which will contribute to the electronic polarization as

$$
\mathbf{P}_{i}=\frac{e}{V} \sum_{j} \Delta \boldsymbol{\tau}_{j i}\left|\xi_{i j}\right|^{2} w_{i j}^{2}
$$


(note that the sign was changed because here we consider the change of the unoccupied band and the electron transfer from the unoccupied $e_{g}$ orbital 5 to the occupied orbitals 1-4).

In order to obtain the total polarization, one should sum up all inequivalent dipoles, induced by the magnetic symmetry breaking. Moreover, it is straightforward to show that

$$
\left|\xi_{i j}\right|^{2}=\frac{1}{2}\left(1+\mathbf{e}_{i} \cdot \mathbf{e}_{j}\right)
$$

Therefore, the spin dependence of $\mathbf{P}_{i}$ in the DE model is given by the isotropic correlation functions, $\mathbf{e}_{i} \cdot \mathbf{e}_{j}$, between directions of spins. This behavior should also specify the temperature dependence of the polarization, associated with the spin disorder.

The spin dependence of $\mathbf{P}$ has the same form as for the phenomenological magnetostriction mechanism,,$\frac{31}{1}$ which is frequently used for the analysis of magnetoelectric (ME) coupling in manganites. $\stackrel{5}{\underline{6}}$ Nevertheless, the new point of our analysis is that this dependence is natural result of the DE physics and is not necessary related to the magnetically driven FE displacements. Formally speaking, the proposed DE mechanism can take place even in a centrosymmetric crystal structure without any magnetostriction, although these two effects can coexist: Once the inversion symmetry is broken by the magnetic order, there will be magnetostrictive forces, which will move the atoms away from their centrosymmetric positions. This will activate the magnetostriction mechanism. In terms of the modern Berry phase theory of polarization, ${ }^{17,18}$ one can say that the DE and magnetostriction mechanism give rise to, respectively, electronic and ionic parts of the polarization. According to the first-principles calculations, the electronic contribution in manganites is at least equally important as the ionic one and cannot be neglected. $\stackrel{4}{*}$

By summarizing this section: in the DE model, the inversion symmetry is broken by noncentrosymmetric modulation of the transfer integrals by $\xi_{i j}$. However, this modulation will also interplay with the crystallographic symmetry of manganites, which is reflected in the behavior of $\Delta \boldsymbol{\tau}_{j i}$ and $\hat{\mathrm{t}}_{i j}$. In the next section, we will illustrate how this interplay will work for different types of manganites, giving rise to finite ME coupling. 


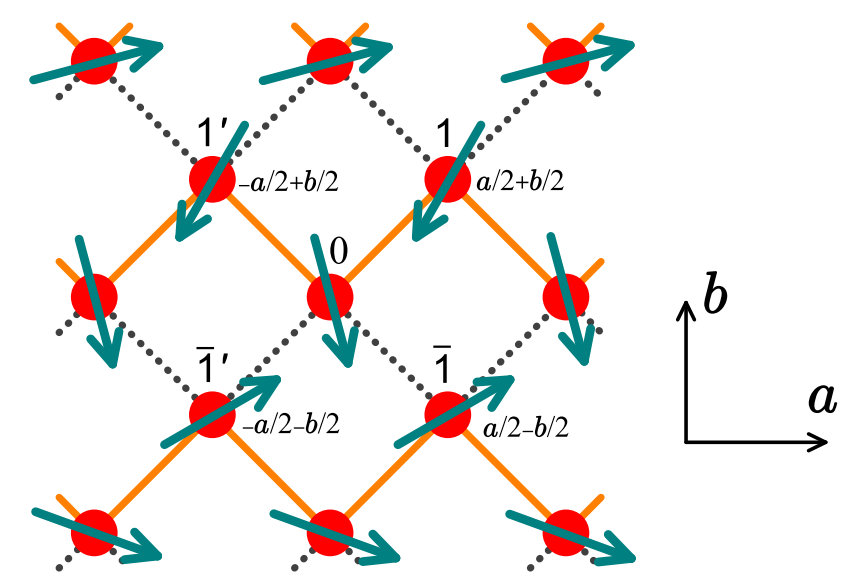

FIG. 2. (Color online) Schematic view on the crystal and magnetic structures in the $\boldsymbol{a} \boldsymbol{b}$ plane of orthorhombic manganites. Mn atoms are indicated by circles. Two types of bonds, which are transformed to themselves by the symmetry operation $\left\{\hat{C}_{a}^{2} \mid \boldsymbol{a} / 2+\boldsymbol{b} / 2\right\}$, are shown by solid and broken lines. The magnetic structure is periodic along the orthorhombic $\boldsymbol{a}$ axis and may have arbitrary periodicity along the $\boldsymbol{b}$ axis.

\section{RESULTS AND DISCUSSIONS}

\section{A. Orthorhombic manganites}

The connection between FE polarization and magnetic structure of orthorhombic (Pbnm) manganites was discussed in our previous article (Ref. 9), also on the level of the DE model. In this section we present a more general and more comprehensive analysis of the problem.

We assume that the manganites form the perfect antiferromagnetic (AFM) order along the orthorhombic $\boldsymbol{c}$ axis, that is indeed consistent with the experimental data. ${ }^{32}$ Then, in the DE model, all $\boldsymbol{a} \boldsymbol{b}$ planes become effectively decoupled, and it is sufficient to consider the single plane. Moreover, it is assumed that the magnetic structure is periodic along the $\boldsymbol{a}$ axis and may have arbitrary periodicity along the $\boldsymbol{b}$ axis (see Fig. 2), being again in total agreement with the experimental situation. $\underline{33}$ The most known examples are the twofold periodic E-type AFM structure, realized in $\mathrm{HoMnO}_{3}$ and $\mathrm{YMnO}_{3},{ }^{25,33,34}$ and the nearly fourfold periodic "spiral" magnetic structure, realized in $\mathrm{TbMnO}_{3} \cdot \underline{32,33,35,36}$ However, it was also suggested that both types of magnetic structures are deformed by relativistic interactions and this deformation has a profound effect on the value of the FE polarization. ${ }^{20,21}$ 
The crystal structure in the $\boldsymbol{a} \boldsymbol{b}$ plane can be generated by two symmetry operation: $\left\{\hat{C}_{a}^{2} \mid \boldsymbol{a} / 2+\boldsymbol{b} / 2\right\}$ (the $180^{\circ}$ rotation around the orthorhombic $\boldsymbol{a}$ axis, combined with the translation by $\boldsymbol{a} / 2+\boldsymbol{b} / 2$ ) and the inversion $\hat{I}$. Moreover, the Mn sites are located in the inversion centers.

Then, we take an arbitrary Mn site ('0' in Fig. 2) and evaluate its contribution to the FE polarization, using Eq. (5). In this case, we should sum up the contributions of four bonds: 0-1, 0-1', 0- $\overline{1}^{\prime}$, and 0-1, which correspond to $\Delta \boldsymbol{\tau}_{j i}=\boldsymbol{a} / 2+\boldsymbol{b} / 2,-\boldsymbol{a} / 2+\boldsymbol{b} / 2,-\boldsymbol{a} / 2-\boldsymbol{b} / 2$, and $\boldsymbol{a} / 2-\boldsymbol{b} / 2$, respectively. The inversion $\hat{I}$ transforms the bonds $0-\overline{1}^{\prime}$ and $0-\overline{1}$ to the bonds $0-1$ and $0-1^{\prime}$, respectively, and the symmetry operation $\left\{\hat{C}_{a}^{2} \mid \boldsymbol{a} / 2+\boldsymbol{b} / 2\right\}$ transforms the bond $0-1^{\prime}$ to the bond 1-0. Hence, we have $w_{0 \overline{1}^{\prime}}=w_{01}, w_{0 \overline{1}}=w_{01^{\prime}}$, and $w_{01^{\prime}}=w_{10}$. Moreover, the periodicity of the magnetic structure along $\boldsymbol{a}$ imposes the following constraints: $\xi_{01^{\prime}}=\xi_{01} \equiv$ $\xi_{0}^{+}$and $\xi_{0 \overline{1}^{\prime}}=\xi_{0 \overline{1}} \equiv \xi_{0}^{-}$, where the notations $\xi_{0}^{+}$and $\xi_{0}^{-}$stand for the bonds spreading in the positive and negative directions of the $\boldsymbol{b}$ axis, starting from the site 0 . Then, the vector $\mathbf{P}_{0}$ can be presented in the form: $\mathbf{P}_{0}=\frac{1}{2 L}\left\{\left|\xi_{0}^{+}\right|^{2}-\left|\xi_{0}^{-}\right|^{2}\right\}\left(P_{0}^{a}, P_{0}^{b}, 0\right)$, where

$$
P_{0}^{a}=\frac{e a}{V_{0}}\left(w_{01}^{2}-w_{10}^{2}\right)
$$

is the FE polarization of the E-type AFM phase $\left(P_{0}^{a} \equiv P_{E}\right), \underline{9}$

$$
P_{0}^{b}=\frac{e a}{V_{0}}\left(w_{01}^{2}+w_{10}^{2}\right) \text {. }
$$

Since all Mn sites are located strictly in the $\boldsymbol{a} \boldsymbol{b}$ plane and, in the DE model, all transfer integrals to the neighboring planes are suppressed by the AFM order, there will be no polarization parallel to the orthorhombic $\boldsymbol{c}$ axis.

Then, we can repeat this procedure and evaluate the contributions associated with the neighboring sites 1 and $\overline{1}$ in the magnetic cell (see Fig. 2). Clearly, half of these contributions will involve the same bonds $0-1,0-1^{\prime}, 0-\overline{1}^{\prime}$, and $0-\overline{1}$. Moreover, since the sites 1 and $\overline{1}$ are obtained from the site 0 by the symmetry operation $\left\{\hat{C}_{a}^{2} \mid \boldsymbol{a} / 2+\boldsymbol{b} / 2\right\}$, in the corresponding expressions for $P_{1}^{a}=P_{\overline{1}}^{a}$ and $P_{1}^{b}=P_{\overline{1}}^{b}$ we will have to interchange $w_{01}$ and $w_{10}$. Furthermore, we note that $\xi_{1}^{-}=\xi_{0}^{+}$and $\xi_{\overline{1}}^{+}=\xi_{0}^{-}$. Then, one can see that $P_{1}^{b}=P_{\overline{1}}^{b}=P_{0}^{b}$. Therefore, all such contributions will be canceled out. On the other hand, the contributions parallel to $\boldsymbol{a}$ will satisfy the condition $P_{1}^{a}=P_{\overline{1}}^{a}=-P_{0}^{a}$. These contributions can be regrouped so that the total polarization parallel to $\boldsymbol{a}$ can be presented in the following form:

$$
P^{a}=\frac{1}{L} \sum_{i=0}^{2 L-1}(-1)^{i}\left|\xi_{i}^{+}\right|^{2} P_{E}
$$


where the summation runs over inequivalent sites of the magnetic unit cell (note also that the crystallographic cell of orthorhombic manganites contains two Mn sites in the $\boldsymbol{a} \boldsymbol{b}$ plane).

Thus, the polarization will be parallel to the $\boldsymbol{a}$ axis. Similar result was obtained in our previous work,$\frac{9}{}$ where we considered the magnetic structures, which respect the symmetry operation $\left\{\hat{C}_{a}^{2} \mid \boldsymbol{a} / 2+\boldsymbol{b} / 2\right\}$. The present work indicates that this result is more general and does not require any specific symmetry of the magnetic structure, apart from its periodicity along the $\boldsymbol{a}$ axis and the AFM coupling along the $\boldsymbol{c}$ axis. Another important point is that the FE polarization in all magnetic structures can be obtained by scaling the one in the E-type AFM state. The scaling factor depends on the relative orientation of spins. Below, we will study it more in details.

Then, Eq. (6) can be rewritten for our purposes as $\left|\xi_{i}^{+}\right|^{2}=\frac{1}{2}\left(1+\mathbf{e}_{i} \cdot \mathbf{e}_{i+1}\right)$. By substituting it in Eq. (8) and noting that only the direction-dependant part of $\left|\xi_{i}^{+}\right|^{2}$ will contribute to $P^{a}$, one can find that

$$
P^{a}=\frac{1}{2 L} \sum_{i=0}^{2 L-1}(-1)^{i} \mathbf{e}_{i} \cdot \mathbf{e}_{i+1} P_{E} .
$$

This formula has the following consequences:

(i) In the perfect E-type AFM structure, $\mathbf{e}_{i} \cdot \mathbf{e}_{i+1}$ is equal to +1 and -1 for the even and odd $i$, respectively. However, in the latter case, the minus sign will be additionally changed due to the prefactor $(-1)^{i}$. Therefore, all terms in the sum will be equal to 1 and we will indeed obtain that $P^{a}=P_{E}$.

One can also consider the deformation of the E-type AFM state, where the odd sublattice is additionally rotated relative to the even one by the angle $\phi$ (see, e.g., Fig. 3 of Ref. 4). Such a deformation is caused by the SO interaction and was obtained in the HF calculations for the realistic Hubbard-type model. ${ }^{20,21}$ In this case, we have $\mathbf{e}_{i} \cdot \mathbf{e}_{i+1}= \pm \cos \phi$ and, therefore,

$$
P^{a}=\cos \phi P_{E}
$$

Thus, for small $\phi$, the first correction to $P_{E}$ appears only in the second order of $\phi$. This explains that the FE polarization in the E-phase is relatively robust against the small canting of spins. However, $P^{a}$ vanishes in the spin-spiral state, corresponding to $\phi=90^{\circ}$.

(ii) The previous claim appears to be more general and can be reformulated as follows: Any homogeneous arrangement of spins, which is characterized by the same values of $\mathbf{e}_{i} \cdot \mathbf{e}_{i+1}$ for all bonds in the $\boldsymbol{a} \boldsymbol{b}$ plane, does not break the inversion symmetry. All these states have 
zero electric polarization, that directly follows from Eq. (9). Such a situation is realized, for instance, in the ferromagnetic (FM) state or in the homogeneous spin-spiral state, despite a widespread believe. $\frac{1}{-}$ Therefore, in order to obtain the finite polarization, it is essential to deform the spin spiral. Let us consider such a deformed spin-spiral structure in the $\boldsymbol{a} \boldsymbol{b}$ plane, for which $\mathbf{e}_{i}=\left(\cos \varphi_{i}, \sin \varphi_{i}, 0\right)$ and $\varphi_{i}=\boldsymbol{q} \cdot \boldsymbol{R}_{i}+\alpha_{i}$. Namely, the phase $\boldsymbol{q} \cdot \boldsymbol{R}_{i}$ describes the propagation of the homogeneous spin spiral and the small parameters $\alpha_{i}$, satisfying the condition $\sum_{i=0}^{2 L-1} \alpha_{i}=0$, describe its deformation. The general geometry of the magnetic structure implies that $\boldsymbol{q}=(0, q, 2 \pi / c)$ and $q b L=2 \pi n$, where $n$ is an integer. Then, since $\mathbf{e}_{i} \cdot \mathbf{e}_{i+1}=\cos \left(\varphi_{i+1}-\varphi_{i}\right)$, we will have

$$
P^{a}=\frac{1}{2 L} \sum_{i=0}^{2 L-1}(-1)^{i} \cos \left(\frac{\pi n}{L}+\alpha_{i+1}-\alpha_{i}\right) P_{E} .
$$

In the first order of $\left(\alpha_{i+1}-\alpha_{i}\right)$, this expression can be further transformed to

$$
P^{a} \approx-\frac{1}{2 L} \sin \left(\frac{\pi n}{L}\right) \sum_{i=0}^{2 L-1}(-1)^{i}\left(\alpha_{i+1}-\alpha_{i}\right) P_{E} .
$$

Thus, the spin-spiral inhomogeneity contributes to $P^{a}$ in the first order of $\left\{\alpha_{i}\right\}$. If the phases $\left\{\alpha_{i}\right\}$ are small, the corresponding polarization is also expected to be small.

(iii) Eq. (7) clearly shows the similarities and differences between the FE polarization and interatomic superexchange interactions. Indeed, the expression $\Delta\left(w_{01}^{2}+w_{10}^{2}\right)$ is nothing but the (minus) energy gain caused by the FM alignment of spins in the bond 0-1, which contributes to the superexchange interaction. $\frac{37}{7}$ Thus, this superexchange interaction is given by the same parameters $w_{01}^{2}$ and $w_{10}^{2}$. The basic difference is that the superexchange interaction is given by the symmetric part of $w_{01}^{2}$ and $w_{10}^{2}$, while the polarization depends on the antisymmetric part.

Below, we present some numerical estimates, using results of previous calculations for the magnetic ground state and FE polarization of orthorhombic manganites. ${ }^{9}, 20,21$ Namely, we take the magnetic structures, obtained in the unrestricted HF calculations with the SO coupling, and analyze the behavior of the FE polarization in terms of the simplified expressions (10) and (11), obtained in the DE model for the deformed magnetic structures of the AFM E-type and spin-spiral type, respectively. In the HF calculations, we typically obtain two types of magnetic structures with the twofold $(L=2)$ and fourfold $(L=4)$ periodicity. For $\mathrm{HoMnO}_{3}$, they are shown in Fig. 3. Similar behavior was obtained for 

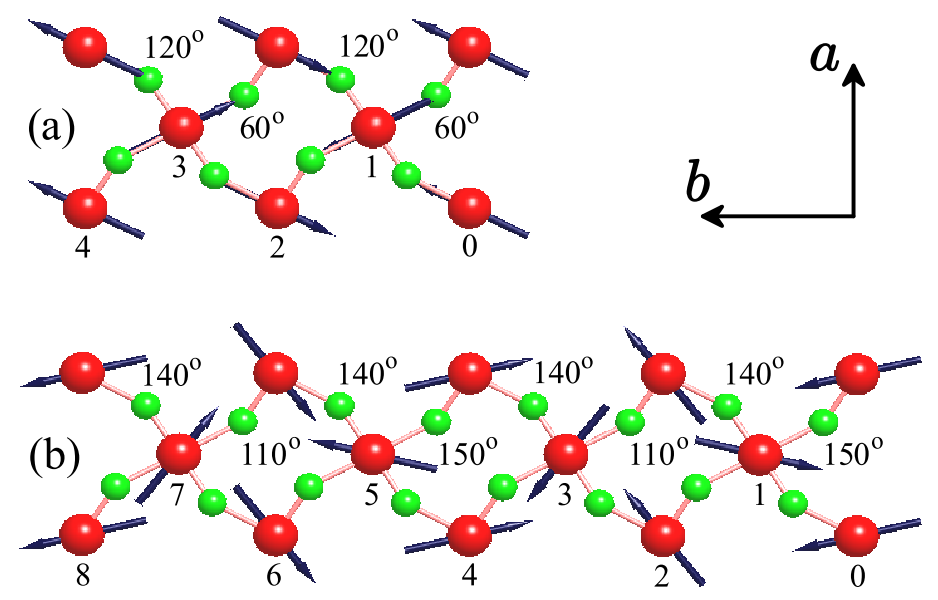

FIG. 3. (Color online) Twofold periodic (a) and fourfold periodic (b) spin structures, as obtained in the mean-filed Hartree-Fock calculations for the effective Hubbard-type model, constructed for the $\mathrm{Pbnm}$ phase of $\mathrm{HoMnO}_{3}$. The manganese atoms are indicated by the big red (dark) spheres and the oxygen atoms are indicated by the small green (grey) spheres. The same numbering of Mn atoms is used in Table I. Other numbers stand for the angles $\left(\varphi_{i+1}-\varphi_{i}\right)$ between spin magnetic moments in the Mn-O-Mn bonds.

$\mathrm{TbMnO}_{3}$ and $\mathrm{YMnO}_{3}{ }^{20,21}$ The magnetic structures with larger periodicity typically include the fragments of low periodic structures and the domain wall-defects (see Fig. 4 of Ref. 20).

As was explained above, the twofold periodic structure can be viewed as the deformed E-type AFM state. The deformation is caused by the single-ion anisotropy. For the Pbnm phases, the angle $\phi$, characterizing this deformation, varies from $63^{\circ}$, in the case of $\mathrm{TbMnO}_{3}, \underset{20}{2}$ till $60^{\circ}$ in the case of $\mathrm{YMnO}_{3}$ and $\mathrm{HoMnO}_{3}$ (Ref. 21 and Fig. 3). Then, $P^{a}$ can be estimated using Eq. (10) as $P^{a} \approx 0.5 P_{E}$, which is perfectly consistent with results of the HF calculations. Indeed, the values of $P_{E}$, obtained in the $\mathrm{HF}$ calculations for the collinear E-type AFM phase without the SO coupling, are 0.96, 1.09, and $1.04 \mu \mathrm{C} / \mathrm{cm}^{2}$ for $\mathrm{TbMnO}_{3}, \mathrm{HoMnO}_{3}$, and $\mathrm{YMnO}_{3}$, respectively. The same calculations, but with the SO coupling, yield $P^{a}=0.47,0.57$, and $0.55 \mu \mathrm{C} / \mathrm{cm}^{2}$ for $\mathrm{TbMnO}_{3}, \mathrm{HoMnO}_{3}$, and $\mathrm{YMnO}_{3}$, respectively. Thus, in all three examples, the above relationship $P^{a} \approx 0.5 P_{E}$ works very well and this tendency is nicely explained by the DE model, where the SO interaction is used in order to obtain the direction of spins in the ground state, while the FE polarization is calculated as a nonrelativistic quantity for the given distribution of spins. 
Similar analysis can be done for the fourfold periodic structure, which can be viewed as a deformed spin spiral, propagating along the $\boldsymbol{b}$ axis. In this case, the spin spiral is stabilized by isotropic magnetic interactions and deformed by anisotropic and DM interactions. ${ }^{20}$ First, we take the angles $\varphi_{i}$, characterizing the directions of spins in the actual HF calculations with the SO coupling (see Fig. 3), and decompose them into the homogeneous $\left(\boldsymbol{q} \cdot \boldsymbol{R}_{i}\right)$ and inhomogeneous $\left(\alpha_{i}\right)$ parts. Then, $(q b / 2)$ is the averaged value of $\left(\varphi_{i+1}-\varphi_{i}\right)$ in the magnetic supercell and $\left(\alpha_{i+1}-\alpha_{i}\right)=\left(\varphi_{i+1}-\varphi_{i}\right)-q b / 2$. For all three compounds, we obtain $q=3 / 4$ (in units of reciprocal lattice translation $g_{b}=2 \pi / b$ ), which is close to the equilibrium values $q \approx 0.68 \div 0.72$, obtained in the spin-spiral calculations without the SO coupling. $\underline{20}$ The parameters $\left(\alpha_{i+1}-\alpha_{i}\right)$, characterizing the deformation of the spin spiral, are summarized in Table I. Then, $P^{a}$ can be estimated using Eq. (11) (note, that $q=3 / 4$ corresponds to

TABLE I. Parameters $\Delta \alpha_{i} \equiv \alpha_{i+1}-\alpha_{i}$, characterizing deformation of the spin-spiral state (in degrees), as obtained in the unrestricted Hartree-Fock calculations with the spin-orbit coupling for the Pbnm phase of $\mathrm{TbMnO}_{3}, \mathrm{HoMnO}_{3}$, and $\mathrm{YMnO}_{3}$. The atomic positions are explained in Fig. 3 ,

\begin{tabular}{lccc}
\hline \hline Parameters & $\mathrm{TbMnO}_{3}$ & $\mathrm{HoMnO}_{3}$ & $\mathrm{YMnO}_{3}$ \\
\hline$\Delta \alpha_{0}=\Delta \alpha_{4}$ & 18 & 15 & 13 \\
$\Delta \alpha_{1}=\Delta \alpha_{5}$ & 7 & 5 & 5 \\
$\Delta \alpha_{2}=\Delta \alpha_{6}$ & -32 & -25 & -23 \\
$\Delta \alpha_{3}=\Delta \alpha_{7}$ & 7 & 5 & 5 \\
\hline \hline
\end{tabular}

$n=3$ ), which yields $P^{a} / P_{E} \approx 0.115,0.081$, and 0.077 for $\mathrm{TbMnO}_{3}, \mathrm{HoMnO}_{3}$ and $\mathrm{YMnO}_{3}$, respectively. These values are well consistent with results of unrestricted HF calculations without additional approximations $\left(P^{a} / P_{E}=0.138,0.110\right.$, and 0.101 for $\mathrm{TbMnO}_{3}, \mathrm{HoMnO}_{3}$ and $\mathrm{YMnO}_{3}$, respectively).

Thus, the FE polarization in the "spin-spiral" phase is about one order of magnitude smaller than in the collinear E-phase, in agreement with the experimental data. $\stackrel{33}{\text { However, }}$ this polarization is caused by the deformation of the spin spiral (and not by the spin-spiral alignment itself). Even in the "spin-spiral" phase, the FE polarization can be obtained by scaling the one of the E-phase, where the scaling factor depends only on the relative directions of spins, and all dependencies on the crystal structure itself are incorporated into $P_{E}$. 


\section{B. Monoclinic $\mathrm{BiMnO}_{3}$}

$\mathrm{BiMnO}_{3}$ is another important compound in the field of multiferroics, and also the most controversial one. It was regarded as a canonical example of multiferroics, where the ferroelectricity indeed coexists with the ferromagnetism, but because of two different mechanisms: the lone pair effect of a nonmagnetic origin was believed to be responsible for the noncentrosymmetric atomic displacements, which simply coexist with the magnetic properties, developed in the Mn sublattice. $\frac{38}{}$ However, this point of view was basically refuted by subsequent experimental studies (Ref. 39) and electronic structure calculations (Ref. 40), which suggest that $\mathrm{BiMnO}_{3}$ should crystallize in the centrosymmetric $C 2 / c$ structure. A "compromised" point of view was proposed in Ref. 22, which suggests that $\mathrm{BiMnO}_{3}$ could be an improper multiferroic, where the inversion symmetry is broken by some hidden AFM order. This magnetic inversion breaking gives rise not only to the FE activity, but also to the DM interactions across the inversion centers, which lead to the FM canting of spins..$^{41}$

The monoclinic $C 2 / c$ phase of $\mathrm{BiMnO}_{3}$ has four formula units (see Fig,4). In the following, it is convenient to work with the fractional coordinates, where each vector $\boldsymbol{v} \equiv$ $\left(v^{1}, v^{2}, v^{3}\right)$ is given in terms of the primitive translations $\boldsymbol{a}_{1}=\frac{1}{2}(a \sin \beta,-b, a \cos \beta), \boldsymbol{a}_{2}=$ $\frac{1}{2}(a \sin \beta, b, a \cos \beta)$, and $\boldsymbol{a}_{3}=(0,0, c)$ as $\boldsymbol{v}=v^{1} \boldsymbol{a}_{1}+v^{2} \boldsymbol{a}_{2}+v^{3} \boldsymbol{a}_{3}$. Then, the Mn sites, which are labeled in the figure as $1,2,3$ and 4 , are located at $(x,-x, 1 / 4),(-x, x,-1 / 4),(1 / 2,0,0)$, and $(0,1 / 2,1 / 2)$, respectively. We use the experimental structure parameters $a=9.529 \AA$, $b=5.604 \AA, c=9.848 \AA, \beta=110.58^{\circ}$, and $x=0.21537$, measured at $4 \mathrm{~K} . \underline{39}$ The $\mathrm{Mn}$ atoms form two inequivalent groups: $(1,2)$ and $(3,4)$. The atoms 1 and 2 can be transformed to each other by the inversion operation $\hat{I}$, while the atoms 3 and 4 are connected by the symmetry operation $\left\{\hat{C}_{y}^{2} \mid \boldsymbol{a}_{3} / 2\right\}$. Therefore, it is sufficient to consider the dipoles around the sites 1 and 3. Similar results for the sites 2 and 4 can be obtained by applying the symmetry operations of the space group $C 2 / c$. Moreover, the atoms in each of the inequivalent groups are surrounded by the atoms from another group.

The crystallographic symmetry can be further lowered by the magnetic order. In this work we consider the scenario where the magnetic group of $\mathrm{BiMnO}_{3}$ has only one nontrivial symmetry operation: $\left\{m_{y} \mid \mathbf{a}_{3} / 2\right\}$ (the mirror reflection $y \rightarrow-y$, combined with the translation by $\left.\mathbf{a}_{3} / 2\right)$. This symmetry was indeed obtained in the previous calculations. ${ }^{22}$ Within each inequivalent subgroups of atoms, $(1,2)$ and $(3,4),\left\{m_{y} \mid \mathbf{a}_{3} / 2\right\}$ transforms the Mn sites to each 


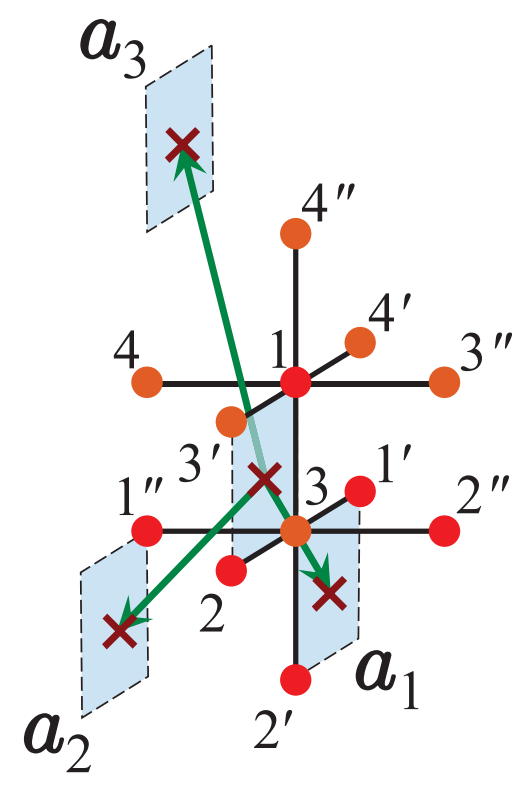

FIG. 4. (Color online) Schematic view on the pseudocubic $C 2 / c$ structure of $\mathrm{BiMnO}_{3}$. There are four Mn sites in the primitive cell, which are labeled as 1, 2, 3 and 4 . These sites form two inequivalent subgroups: $(1,2)$ and $(3,4)$, which are shown by different colors. Other atoms, located in the nearest neighborhood of the inequivalent sites 1 and 3 , are obtained by the primitive translations $\boldsymbol{a}_{1}, \boldsymbol{a}_{2}$, and $\boldsymbol{a}_{3}$. The inversion centers (marked by the symbols $\times$ ) are located in the centers of the distorted cube face, formed by the atoms 1,2 , and two atoms of the type either 3 or 4 (for instance $1-3-2-3^{\prime}$ in the figure).

other. Therefore, if $\mathbf{e}_{1}=\left(e_{1}^{x}, e_{1}^{y}, e_{1}^{z}\right)$ is the direction of spin at the site 1 , the one at the site 2 will be given by $\mathbf{e}_{2}=\left(-e_{1}^{x}, e_{1}^{y},-e_{1}^{z}\right)$ (note that $\mathbf{e}$ is the axial vector, and here $x, y$, and $z$ denote the directions in the monoclinic frame). Similar property holds for $\mathbf{e}_{3}$ and $\mathbf{e}_{4}$. Thus, $y$-projections of spins are ordered ferromagnetically, while the $x$ - and $z$-projections are ordered antiferromagnetically. Then, we will have the following symmetry properties: $\mathbf{e}_{1} \cdot \mathbf{e}_{4}=\mathbf{e}_{2} \cdot \mathbf{e}_{3}=e_{1}^{y} e_{3}^{y}-\mathbf{e}_{1}^{\perp} \cdot \mathbf{e}_{3}^{\perp}$ and $\mathbf{e}_{2} \cdot \mathbf{e}_{4}=\mathbf{e}_{1} \cdot \mathbf{e}_{3}$, where $\mathbf{e}^{\perp}$ is the AFM component of spin being perpendicular to $y$. Thus, if $e_{i}^{x}=e_{i}^{y}=0$, we obtain the so-called $\uparrow \downarrow \uparrow \downarrow$ AFM phase, which breaks the inversion symmetry. ${ }^{22}$ If $e_{i}^{x}=e_{i}^{z}=0$, we deal with the regular FM phase, which preserves the inversion symmetry.

First, let us consider the contribution of the site 1 to the FE polarization. It has six nearest neighbors: $3,3^{\prime}, 3^{\prime \prime}, 4,4^{\prime}$, and $4^{\prime \prime}$, which are located at $(1 / 2,0,0),(-1 / 2,0,0)$, 
$(1 / 2,-1,0),(0,1 / 2,1 / 2),(1,-1 / 2,1 / 2)$, and $(0,-1 / 2,1 / 2)$, respectively. Then, Eq.(55) will yield

$$
\begin{aligned}
\mathbf{P}_{1} & =\left|\xi_{13}\right|^{2} \frac{e}{V}\left(w_{13}^{2} \Delta \boldsymbol{\tau}_{31}+w_{13^{\prime}}^{2} \Delta \boldsymbol{\tau}_{3^{\prime} 1}+w_{13^{\prime \prime}}^{2} \Delta \boldsymbol{\tau}_{3^{\prime \prime} 1}\right) \\
& +\left|\xi_{14}\right|^{2} \frac{e}{V}\left(w_{14^{\prime \prime}}^{2} \Delta \boldsymbol{\tau}_{4^{\prime \prime} 1}+w_{14}^{2} \Delta \boldsymbol{\tau}_{41}+w_{14^{\prime}}^{2} \Delta \boldsymbol{\tau}_{4^{\prime} 1}\right) .
\end{aligned}
$$

The symmetry operation $\left\{\hat{C}_{y}^{2} \mid \boldsymbol{a}_{3} / 2\right\}$ transforms the site 1 to itself, and the sites $3,3^{\prime}$, and $3^{\prime \prime}$ to the sites $4^{\prime \prime}, 4$, and $4^{\prime}$, respectively. Therefore, we will have the following properties: $w_{14^{\prime \prime}}^{2}=w_{13}^{2}, w_{14}^{2}=w_{13^{\prime}}^{2}$, and $w_{14^{\prime}}^{2}=w_{13^{\prime \prime}}^{2}$. Similar expression for $\mathbf{P}_{2}$ can be obtained by applying the inversion operation and replacing $\xi_{13}$ and $\xi_{14}$ by $\xi_{23}$ and $\xi_{24}$, respectively. Then, using the symmetry properties of $w_{i j}^{2}$ and $\left|\xi_{i j}\right|^{2}$, together with Eq. (6) , and noting that $\Delta \boldsymbol{\tau}_{31}-\Delta \boldsymbol{\tau}_{4^{\prime \prime} 1}=\Delta \boldsymbol{\tau}_{34^{\prime \prime}} \equiv(1 / 2,1 / 2,-1 / 2)$ and $\Delta \boldsymbol{\tau}_{3^{\prime} 1}-\Delta \boldsymbol{\tau}_{41}=\Delta \boldsymbol{\tau}_{3^{\prime \prime} 1}-\Delta \boldsymbol{\tau}_{4^{\prime} 1}=\Delta \boldsymbol{\tau}_{3^{\prime} 4} \equiv$ $(-1 / 2,-1 / 2,-1 / 2)$, one can find that

$$
\mathbf{P}_{1}+\mathbf{P}_{2}=\mathbf{e}_{1}^{\perp} \cdot \mathbf{e}_{3}^{\perp} \frac{e}{V}\left(w_{13}^{2} \Delta \boldsymbol{\tau}_{34^{\prime \prime}}+\left[w_{13^{\prime}}^{2}+w_{13^{\prime \prime}}^{2}\right] \Delta \boldsymbol{\tau}_{3^{\prime} 4}\right) .
$$

Similar analysis can be performed for the sites 3 and 4 . For example, the nearest neighbors of the site 3 are: 1, $1^{\prime}, 1^{\prime \prime}, 2,2^{\prime}$, and $2^{\prime \prime}$, which are located at $(x,-x, 1 / 4)$, $(1+x,-x, 1 / 4),(x, 1-x, 1 / 4),(-x, x,-1 / 4),(1-x, x,-1 / 4)$, and $(1-x, x-1,-1 / 4)$, respectively (see Fig. (4). Moreover, the bonds $3-1^{\prime}$ and $3-1^{\prime \prime}$ can be transformed by regular translations to the bonds $3^{\prime}-1$ and $3^{\prime \prime}-1$, respectively. The bonds $3-2,3-2^{\prime}$, and $3-2^{\prime \prime}$ can be transformed to the bonds $3^{\prime}-1,3-1$, and $3^{\prime \prime}-1$, respectively, by combining the inversion operation with appropriate translations. Therefore, we will have the following symmetry properties: $w_{32}^{2}=w_{3^{\prime} 1}^{2}, w_{32^{\prime}}^{2}=w_{31}^{2}, w_{32^{\prime \prime}}^{2}=w_{3^{\prime \prime} 1}^{2}, \Delta \boldsymbol{\tau}_{13}=-\Delta \boldsymbol{\tau}_{2^{\prime} 3}=-\Delta \boldsymbol{\tau}_{31}, \Delta \boldsymbol{\tau}_{1^{\prime} 3}=-\Delta \boldsymbol{\tau}_{23}=-\Delta \boldsymbol{\tau}_{3^{\prime} 1}$, and $\Delta \boldsymbol{\tau}_{1^{\prime \prime} 3}=-\Delta \boldsymbol{\tau}_{2^{\prime \prime 3}}=-\Delta \boldsymbol{\tau}_{3^{\prime \prime 1}}$, which yield

$$
\mathbf{P}_{3}=\left(\left|\xi_{32}\right|^{2}-\left|\xi_{31}\right|^{2}\right) \frac{e}{V}\left(w_{31}^{2} \Delta \boldsymbol{\tau}_{31}+w_{3^{\prime} 1}^{2} \Delta \boldsymbol{\tau}_{3^{\prime} 1}+w_{3^{\prime \prime} 1}^{2} \Delta \boldsymbol{\tau}_{3^{\prime \prime} 1}\right)
$$

Similar expression for the site 4 is obtained by applying the symmetry operation $\left\{\hat{C}_{y}^{2} \mid \boldsymbol{a}_{3} / 2\right\}$, replacing $\xi_{32}$ and $\xi_{31}$ by $\xi_{42}$ and $\xi_{41}$, respectively, and using the same symmetry properties, which were used for derivation of Eq. (12). Then, one can obtain that

$$
\mathbf{P}_{3}+\mathbf{P}_{4}=-\mathbf{e}_{1}^{\perp} \cdot \mathbf{e}_{3}^{\perp} \frac{e}{V}\left(w_{31}^{2} \Delta \boldsymbol{\tau}_{34^{\prime \prime}}+\left[w_{3^{\prime} 1}^{2}+w_{3^{\prime \prime} 1}^{2}\right] \Delta \boldsymbol{\tau}_{3^{\prime} 4}\right) .
$$

Thus, the contribution $\left(\mathbf{P}_{3}+\mathbf{P}_{4}\right)$ has the same form as $\left(\mathbf{P}_{1}+\mathbf{P}_{2}\right)$, but with the opposite sign and reversed order of site indices in all of $w_{i j}^{2}$. 
Then, the total polarization $\mathbf{P}=\sum_{i=1}^{4} \mathbf{P}_{i}$ can be obtained by scaling the one in the $\uparrow \downarrow \uparrow \downarrow$ AFM phase:

$$
\mathbf{P}=\mathbf{e}_{1}^{\perp} \cdot \mathbf{e}_{3}^{\perp} \mathbf{P}_{\uparrow \uparrow \uparrow \downarrow},
$$

where, in the fractional coordinate frame:

$$
P_{\uparrow \uparrow \uparrow \downarrow}^{1}=P_{\uparrow \uparrow \uparrow \downarrow}^{2}=-\frac{e}{2 V}\left(\left[w_{13^{\prime}}^{2}-w_{3^{\prime} 1}^{2}\right]+\left[w_{13^{\prime \prime}}^{2}-w_{3^{\prime \prime} 1}^{2}\right]-\left[w_{13}^{2}-w_{31}^{2}\right]\right),
$$

and

$$
P_{\uparrow \uparrow \uparrow \downarrow}^{3}=-\frac{e}{2 V}\left(\left[w_{13^{\prime}}^{2}-w_{3^{\prime} 1}^{2}\right]+\left[w_{13^{\prime \prime}}^{2}-w_{3^{\prime \prime} 1}^{2}\right]+\left[w_{13}^{2}-w_{31}^{2}\right]\right) .
$$

It corresponds to $\mathbf{P}_{\uparrow \uparrow \uparrow \downarrow}=\left(P_{\uparrow \downarrow \uparrow \downarrow}^{x}, 0, P_{\uparrow \uparrow \uparrow \downarrow}^{z}\right)$ in the cartesian coordinate frame, where

$$
\begin{gathered}
P_{\uparrow \uparrow \uparrow \downarrow}^{x}=\frac{2 V}{b c} P_{\uparrow \uparrow \uparrow \downarrow}^{1}, \\
P_{\uparrow \downarrow \uparrow \downarrow}^{z}=2 V \cot \beta P_{\uparrow \uparrow \uparrow \downarrow}^{x}+\frac{2 V \sec \beta}{a b} P_{\uparrow \downarrow \uparrow \downarrow}^{3},
\end{gathered}
$$

and the primitive cell volume is $V=\frac{1}{2} a b c \sin \beta$.

Therefore, the properties of the multiferroic phase of $\mathrm{BiMnO}_{3}$ can be rationalized as follows:

(i) $\mathbf{P}$ is proportional to the "correlator" $\mathbf{e}_{1}^{\perp} \cdot \mathbf{e}_{3}^{\perp}$, constructed from the AFM components of the neighboring spins. Therefore, by enforcing the FM alignment of spins along the $y$ axis (e.g., by applying an external magnetic field), one can decrease $\mathbf{e}_{1}^{\perp} \cdot \mathbf{e}_{3}^{\perp}$ and, therefore, P. Such a possibility was indeed investigated in the mean-field HF calculations with the external magnetic field $\underline{\underline{22}}$

(ii) Reversing the AFM components of spins at the sites 3 and $4\left(\mathbf{e}_{3}^{\perp} \rightarrow-\mathbf{e}_{3}^{\perp}\right)$, one can flip the direction of polarization $\mathbf{P} \rightarrow-\mathbf{P}$. Particularly, two kinds of the AFM domains, $\uparrow \downarrow \uparrow \downarrow$ and $\uparrow \downarrow \downarrow \uparrow$, should have opposite polarization: $\mathbf{P}_{\uparrow \downarrow \uparrow \downarrow}=-\mathbf{P}_{\uparrow \downarrow \downarrow \uparrow}$. Moreover, by making the AFM projections of spins at the sites 1 and 3 to be orthogonal to each other, one can switch off the polarization.

(iii) Using the values $\mathbf{P}=(-0.611,0,-0.042) \mu \mathrm{C} / \mathrm{cm}^{2}$, obtained in mean-field $\mathrm{HF}$ calculations for the noncollinear magnetic ground state with SO coupling, and $\mathbf{P}_{\uparrow \uparrow \uparrow \downarrow}=$ $(-0.790,0,-0.052) \mu \mathrm{C} / \mathrm{cm}^{2}$, obtained in the same calculations for the collinear $\uparrow \downarrow \uparrow \downarrow A F M$ state without $\mathrm{SO}$ coupling, $\frac{22}{=}$ one can estimate the ratios $P^{x} / P_{\uparrow \uparrow \uparrow \downarrow}^{x}$ and $P^{z} / P_{\uparrow \uparrow \uparrow \downarrow}^{z}$ as 0.773 and 0.808 , respectively. They are well consistent with the value of $\mathbf{e}_{1}^{\perp} \cdot \mathbf{e}_{3}^{\perp}=0.764$, obtained for the noncollinear magnetic ground state. $\frac{22}{\underline{2}}$ Thus, the scaling relation (13) indeed works 
very well and provides a good estimate for the FE polarization in the noncollinear magnetic ground state of $\mathrm{BiMnO}_{3}$.

(iv) $\mathbf{P}_{\uparrow \downarrow \uparrow \downarrow}$ can be estimated using the Wannier weights, $w_{i j}^{2}$, collected in Table II. The details are summarized in Supplemental Materials. .2 Particularly, such an analysis allows us

TABLE II. Weights of Wannier functions, $w_{i j}^{2}$, spreading from the site $i$ to the neighboring site $j$ in $\mathrm{BiMnO}_{3}$. All parameters are dimensionless, in units of $10^{-3}$. The atomic positions are explained in Figs. 4 and 5 .

\begin{tabular}{lcr}
\hline \hline$i j$ & $w_{i j}^{2}$ & $w_{j i}^{2}$ \\
\hline 13 & 0.093 & 13.408 \\
$13^{\prime}$ & 3.801 & 5.318 \\
$13^{\prime \prime}$ & 13.851 & 0.060 \\
\hline \hline
\end{tabular}

to understand why the $z$ component of the polarization is expected to be much weaker than the $x$ one.

First, we note that the contribution of $\left[w_{13^{\prime}}^{2}-w_{3^{\prime} 1}^{2}\right]$ is relatively small. Moreover, the contributions of $\left[w_{13}^{2}-w_{31}^{2}\right]$ and $\left[w_{13^{\prime \prime}}^{2}-w_{3^{\prime \prime} 1}^{2}\right]$ have opposite sign. Therefore, if for $P_{\uparrow \downarrow \uparrow \downarrow}^{3}$ there will be a strong cancelation of these two terms, for $P_{\uparrow \downarrow \uparrow \downarrow}^{1}$ they will collaborate - see Eqs. (14) and (15). Thus, $P_{\uparrow \downarrow \uparrow \downarrow}^{3}$ is expected to be much weaker than $P_{\uparrow \downarrow \uparrow \downarrow}^{1}$. This behavior is closely related to the orbital ordering in the bonds $1-3,1-3^{\prime}$, and $1-3^{\prime \prime}$ (and in the equivalent to them bonds 1-4", 1-4, and 1-4' - see Fig. 51). For example, the overlap between the unoccupied $x^{2}-y^{2}$ orbital at the site 1 and the occupied $3 y^{2}-r^{2}$ orbital at the site 3 is small, that explains the small value of $w_{13}^{2}$. On the other hand, the overlap between unoccupied $z^{2}-x^{2}$ orbital at the site 3 and occupied $3 z^{2}-r^{2}$ orbital at the site 1 is much larger, so as the value of $w_{31}^{2}$. Therefore, we have $w_{13}^{2} \ll w_{31}^{2}$. The situation in the bond 1-3" is exactly the opposite and $w_{13^{\prime \prime}}^{2} \gg w_{3^{\prime \prime} 1}^{2}$. In the bond $1-3^{\prime}$, the overlap between the occupied and unoccupied orbitals is approximately the same in the both directions and $w_{13^{\prime}}^{2} \approx w_{3^{\prime} 1}^{2}$. If such orbital ordering were realized in the ideal cubic lattice, one could use the regular Slater-Koster parametrization for the transfer integrals, ${ }^{43}$ which would yield $\left[w_{13^{\prime \prime}}^{2}-w_{3^{\prime \prime} 1}^{2}\right]=-\left[w_{13}^{2}-w_{31}^{2}\right],\left[w_{13^{\prime}}^{2}-w_{3^{\prime} 1}^{2}\right]=0$, and $P_{\uparrow \downarrow \uparrow \downarrow}^{3}=0$. Therefore, the second term in Eq. (17) will vanish. Moreover, the first term in Eq. (17) will also vanish in the ideal cubic lattice, corresponding to $\beta=90^{\circ}$. Thus, the small value of $P_{\uparrow \downarrow \uparrow \downarrow}^{z}$ can be regarded as the measure of deviation from the perfect cubic 

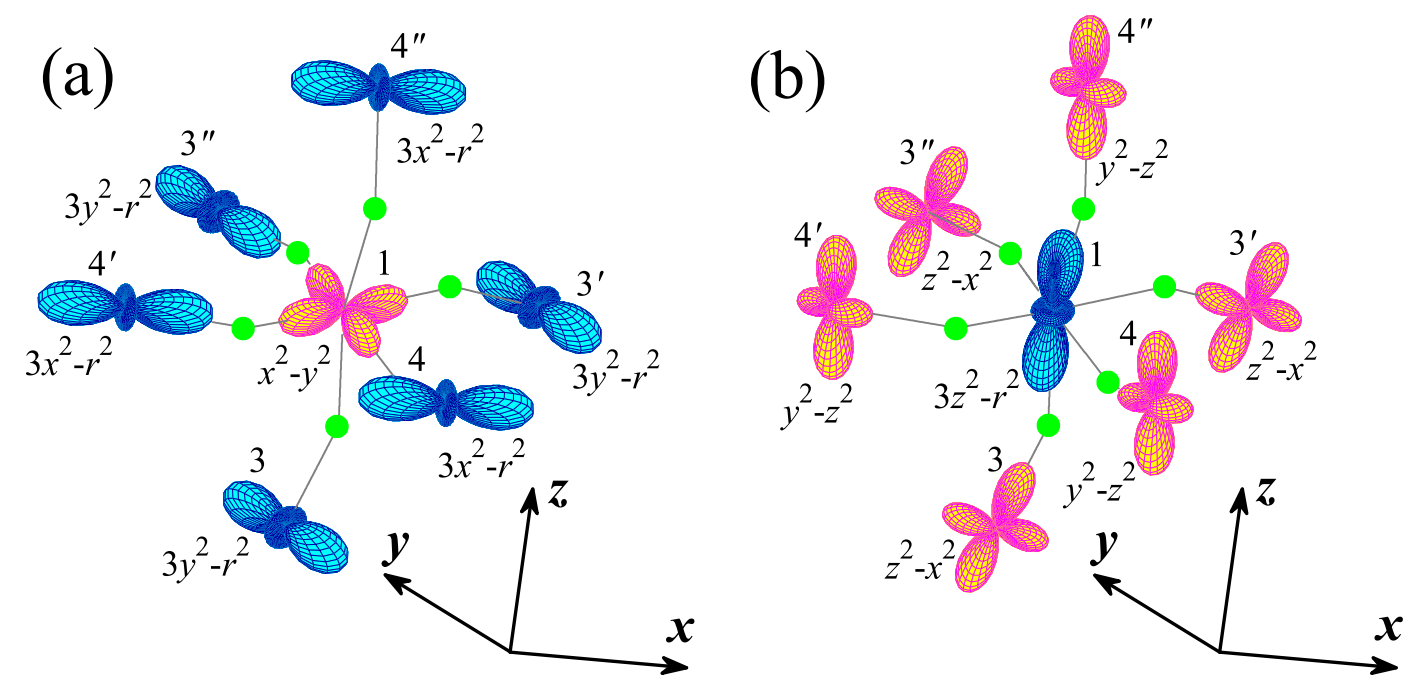

FIG. 5. (Color online) Details of orbital ordering in $\mathrm{BiMnO}_{3}$. (a) The unoccupied $e_{g}$ orbital at the central Mn site 1 together with the occupied $e_{g}$ orbitals at surrounding it Mn sites 3 and 4 . (b) The occupied $e_{g}$ orbital at the site 1 and the unoccupied $e_{g}$ orbital at sites 3 and 4 . The oxygen atoms are indicated by the green (gray) spheres. The notations of orbitals are only approximate ones and correspond to the perfect cubic environment. Here, the axes $x, y$, and $z$ specify the pseudocubic coordinate frame (should not be confused with the directions $x, y$, and $z$ in the monoclinic frame, which are used for the directions of magnetic moment and the symmetry operations of the space group $C 2 / c)$.

environment. On the contrary, $P_{\uparrow \downarrow \uparrow \downarrow}^{x}$ can be finite even in the perfect cubic environment, provided that it supports the specific type of the orbital ordering, shown in Fig. 5. In this case, $P_{\uparrow \downarrow \uparrow \downarrow}^{x}$ is given by the simplified expression $P_{\uparrow \downarrow \uparrow \downarrow}^{x}=\frac{2 e}{b c}\left[w_{13}^{2}-w_{31}^{2}\right]$, where $w_{13}^{2}$ and $w_{31}^{2}$ can be obtained using the Slater-Koster parametrization for the ideal cubic lattice. ${ }^{43}$

Using the values of $w_{i j}^{2}$ reported in Table $\amalg$, the $x$ component of the polarization can be estimated as $P_{\uparrow \downarrow \uparrow \downarrow}^{x}=-0.743 \mu \mathrm{C} / \mathrm{cm}^{2}$, which is in excellent agreement with the value -0.790 $\mu \mathrm{C} / \mathrm{cm}^{2}$, obtained in the HF calculations without additional approximations. However, the agreement for $P_{\uparrow \downarrow \uparrow \downarrow}^{z}$ is not so good: $0.230 \mu \mathrm{C} / \mathrm{cm}^{2}$ in the present model against -0.052 $\mu \mathrm{C} / \mathrm{cm}^{2}$ in the $\mathrm{HF}$ calculations. In the present model analysis, the first term in Eq. (17) clearly dominates. Then, since $\cot \beta<0$, the sign of the first term in $P_{\uparrow \downarrow \uparrow \downarrow}^{z}$ should be opposite to $P_{\uparrow \downarrow \uparrow \downarrow}^{x}$. This contribution should be compensated by the second term in Eq. (17). However, in the present model analysis, the latter term is found to be small. Apparently, 


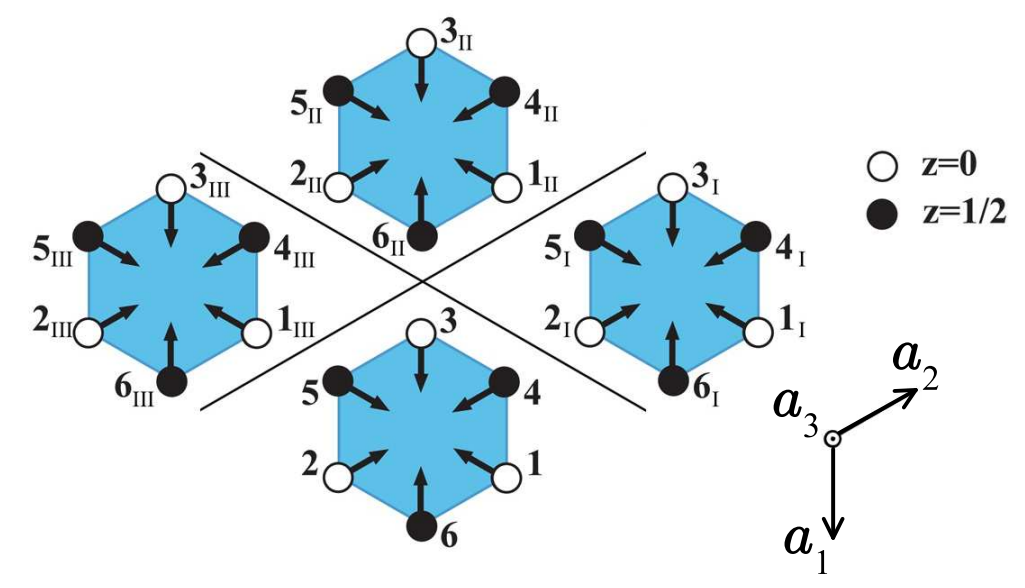

FIG. 6. (Color online) Relative positions of manganese atoms in the hexagonal $P 6_{3} \mathrm{~cm}$ structure. The atoms located in the $z=0$ and $z=1 / 2$ planes are indicated by white and black spheres, respectively. Neighboring unit cells are denoted with Greek numerals. The directions of spins in the magnetic phase $\Gamma_{2}$ are shown by arrows.

there are still some contributions, which are missing in the model and which contribute to the small $z$ component of polarization in the $\uparrow \downarrow \uparrow \downarrow$ phase.

\section{Hexagonal manganites}

The hexagonal $\mathrm{P}_{3} \mathrm{~cm}$ phase of manganites contains six formula units. By choosing the primitive translations as $\boldsymbol{a}_{1}=(0,-a, 0), \boldsymbol{a}_{2}=\left(\frac{\sqrt{3}}{2} a, \frac{1}{2} a, 0\right)$, and $\boldsymbol{a}_{3}=(0,0, c)$ (see Fig. 6 ), the positions of six Mn atoms, in the fractional coordinates, are $(x, x, 0),(0,-x, 0),(-x, 0,0)$, $(0, x, 1 / 2),(-x,-x, 1 / 2)$ and $(x, 0,1 / 2)$. The experimental structure parameters for $\mathrm{YMnO}_{3}$ at $10 \mathrm{~K}$, which we consider as an example, are $a=6.120 \AA, c=11.407 \AA$, and $x=0.3423$ (see Supplementary Information of Ref. 44). All atomic positions can be generated from the first one by applying the $60^{\circ}$-degree rotation around the $z$ axis, combined with the half of the hexagonal translation, $\left\{C_{z}^{6} \mid \mathbf{c} / 2\right\}$.

There are six possible magnetic structures, which are compatible with the space group $P 6_{3} \mathrm{~cm}$ (see Fig. 7) $\stackrel{45,46}{4}$ Among them, the structures $\Gamma_{1}, \Gamma_{4}, \Gamma_{5}$ with $\mathbf{e}_{1} \|$ [120], and $\Gamma_{6}$ with $\mathbf{e}_{1} \|$ [120] differ from, respectively, $\Gamma_{2}, \Gamma_{3}, \Gamma_{5}$ with $\mathbf{e}_{1} \|$ [100], and $\Gamma_{6}$ with $\mathbf{e}_{1} \|[100]$ by the directions of the easy axes, which are controlled by the single-ion anisotropy: ${ }^{23}$ Thus, the 


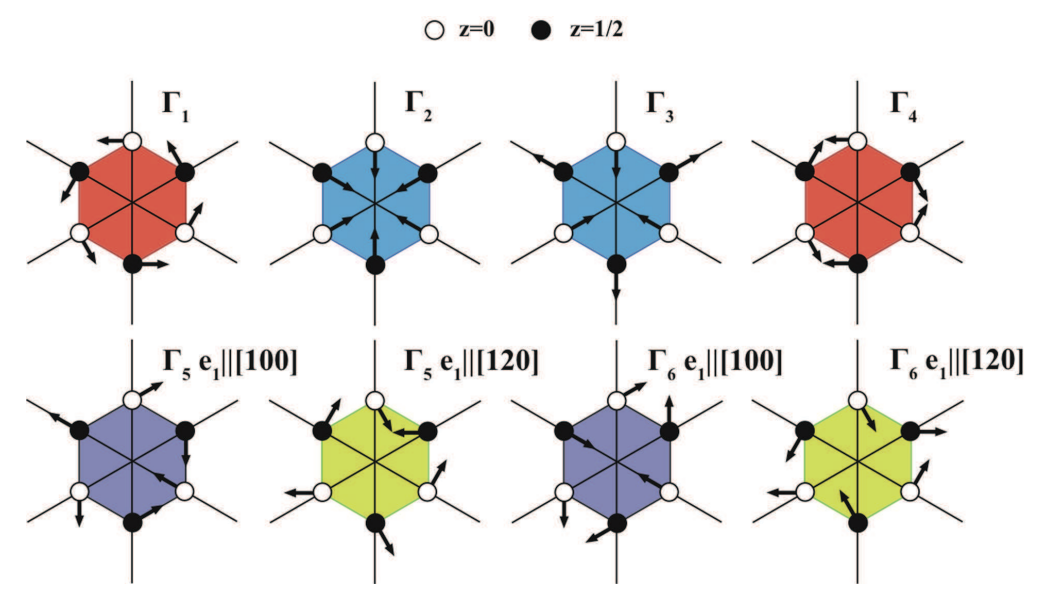

FIG. 7. (Color online) Possible magnetic structures, compatible with the space group $P 6_{3} \mathrm{~cm}$.

difference is of a relativistic origin. Since we do not consider explicitly the relativistic effects, we can treat these two groups of states as equivalent. Moreover, the states $\Gamma_{3}$ and $\Gamma_{5}$ differ from, respectively, $\Gamma_{2}$ and $\Gamma_{6}$ by the magnetic alignment in adjacent $x y$ planes: $\left\{C_{z}^{6} \mid \mathbf{c} / 2\right\}$ transforms $\Gamma_{2}$ as the normal symmetry operation, while in $\Gamma_{3}$, it is additionally combined with the time-reversal operation $\hat{T}$, which additionally flips the spins in every second $x y$ plane. For the $\Gamma_{5}$ and $\Gamma_{6}$ states, the symmetry operation $\left\{C_{z}^{6} \mid \mathbf{c} / 2\right\}$ is additionally combined with the $120^{\circ}$ rotation of spin around the $z$ axis.

Although these materials have no inversion symmetry and are ferroelectric irrespectively on their magnetic structure, the transition from the first group of states $\left(\Gamma_{3}\right.$ and $\left.\Gamma_{5}\right)$ to the second one $\left(\Gamma_{2}\right.$ and $\left.\Gamma_{6}\right)$ is characterized by the finite change of the polarization (about $-120 \mu \mathrm{C} / \mathrm{m}^{2}$, according to the $\mathrm{HF}$ calculations for the effective Hubbard-type model) $\underline{\underline{2}}$ Moreover, the states $\Gamma_{2}$ and $\Gamma_{6}$ are weakly ferromagnetic, giving an interesting possibility for the mutual control of ferroelectricity and magnetism. In this section we will elucidate the microscopic origin of the magnetic state dependence of the FE polarization.

In the following, we assume that all spins lie in the $x y$ plane, and the angles between neighboring spins in the same plane are fixed and equal to either $120^{\circ}$ or $-120^{\circ}$, depending on the type of the magnetic structure. Then, we consider a continuous transformation from $\Gamma_{3}\left(\Gamma_{5}\right)$ to $\Gamma_{2}\left(\Gamma_{6}\right)$, where all the spins in every second $x y$ plane are additionally rotated by the angle $\phi$, varying from $0^{\circ}$ till $180^{\circ}$.

Since relative directions of spins in each $x y$ plane are fixed, there will be no in-plane 
contributions to the spin-dependent part of the polarization and we can go directly to the analysis of inter-plane contributions. First, let us consider the 'dipole', associated with the central site 3 and caused by the transfer of the weight of the Wannier functions to the nearest neighbors in the plane, located in the positive direction of $z$. The corresponding contribution to the spin-dependent part of the polarization can be written as

$$
\mathbf{P}_{3}^{+}=\frac{e}{2 V}\left(\cos (\phi-\alpha) w_{34}^{2} \Delta \boldsymbol{\tau}_{43}+\cos (\phi+\alpha) w_{35}^{2} \Delta \boldsymbol{\tau}_{53}+\cos \phi w_{36_{\mathrm{II}}}^{2} \Delta \boldsymbol{\tau}_{6_{\mathrm{II}} 3}\right)
$$

(see Figs. 6 and Fig. 7 for the notations of atomic sites and the relative directions of spin moments, respectively), where $\alpha=120^{\circ}\left(-120^{\circ}\right)$ for $\Gamma_{3}\left(\Gamma_{5}\right)$. Moreover, due to the mirror reflection $x \rightarrow-x$, which is one of the symmetry operations of the space group $P 6_{3} \mathrm{~cm}$, the bonds 3-4 and 3-5 are equivalent. Therefore, $w_{34}^{2}=w_{35}^{2}$ and the above expression can be further rearranged as

$$
\mathbf{P}_{3}^{+}=\frac{e}{2 V}\left(\cos \phi \cos \alpha w_{34}^{2}\left(\Delta \boldsymbol{\tau}_{43}+\Delta \boldsymbol{\tau}_{53}\right)+\sin \phi \sin \alpha w_{34}^{2} \Delta \boldsymbol{\tau}_{45}+\cos \phi w_{36_{\mathrm{II}}}^{2} \Delta \boldsymbol{\tau}_{6_{\mathrm{II}} 3}\right),
$$

where, in the fractional coordinates, $\Delta \boldsymbol{\tau}_{43}+\Delta \boldsymbol{\tau}_{53}=(x, 0,1), \Delta \boldsymbol{\tau}_{45}=(x, 2 x, 0)$, and $\Delta \boldsymbol{\tau}_{6_{\text {II } 3}}=$ $(2 x-1,0,1 / 2)$. Similar expressions for other sites can be obtained by applying the symmetry operation $\left\{C_{z}^{6} \mid \mathbf{c} / 2\right\}$ to $\mathbf{P}_{3}^{+}$. Then, it is clear that, due to the symmetry, all $x y$ contributions to the total $\mathbf{P}^{+}$, obtained after the summation over six Mn sites in the primitive cell, will be canceled out, and $\mathbf{P}^{+}$will be parallel to $z$. It is given by the following expression

$$
\mathbf{P}^{+}=\frac{3 e}{2 V} \cos \phi\left(w_{36_{\mathrm{II}}}^{2}-w_{34}^{2}\right) \boldsymbol{a}_{3},
$$

which holds for both transitions: from $\Gamma_{3}$ to $\Gamma_{2}$ and from $\Gamma_{5}$ to $\Gamma_{6}$. Similar expression for $\mathbf{P}^{-}$, caused by the transfer of the Wannier weight to the nearest neighbor sites $\overline{4}$ and $\overline{6}_{\text {II }}$, located in the negative direction of $z$, is obtained by replacing $\boldsymbol{a}_{3}$ by $-\boldsymbol{a}_{3}$. Moreover, due to the symmetry operation $\left\{C_{z}^{6} \mid \mathbf{c} / 2\right\}$, the Wannier weights obey the following properties: $w_{3 \overline{6}_{\mathrm{II}}}^{2}=w_{6_{\mathrm{II}} 3}^{2}$ and $w_{3 \overline{4}}^{2}=w_{43}^{2}$. Altogether, this leads to

$$
\mathbf{P}^{-}=-\frac{3 e}{2 V} \cos \phi\left(w_{6_{\mathrm{II}} 3}^{2}-w_{43}^{2}\right) \boldsymbol{a}_{3}
$$

Thus, the dependence of the polarization on the relative directions of spins between neighboring planes obeys the simplest $\cos \phi$ law. The nearest-neighbors (NN) contribution to the polarization change $\Delta \mathbf{P}=\mathbf{P}\left(\Gamma_{2,6}\right)-\mathbf{P}\left(\Gamma_{3,5}\right)$, associated with the transition from $\Gamma_{3}$ and $\Gamma_{5}(\phi=0)$ to, respectively, $\Gamma_{2}$ and $\Gamma_{6}\left(\phi=180^{\circ}\right)$, is given by

$$
\Delta \mathbf{P}_{N N}=\frac{3 e}{V}\left(w_{34}^{2}-w_{43}^{2}-w_{36_{\mathrm{II}}}^{2}+w_{6_{\mathrm{II}} 3}^{2}\right) \boldsymbol{a}_{3}
$$


Similar expression, associated with the transfer of the Wannier weights to the next-nearestneighbor $(\mathrm{NNN})$ sites in the planes $z= \pm 1 / 2$ is obtained by replacing the sites 4 and 6 II by the sites $4_{\text {III }}$ and 6 , respectively (see Fig. 6).

The Wannier weights, $w_{i j}^{2}$, obtained for $\mathrm{YMnO}_{3}$ are collected in Table III, Details can be found in Supplemental Materials. $\underline{\underline{42}}$ Using these parameters, the nearest-neighbor contribu-

TABLE III. Weights of Wannier functions, $w_{i j}^{2}$, spreading from the site $i$ to the site $j$ in the hexagonal $\mathrm{YMnO}_{3}$. All parameters are dimensionless, in units of $10^{-4}$. The atomic positions are explained in Fig. 6. The first two lines show the data for the inequivalent nearest-neighbor (NN) bonds and the second two lines - to the next-nearest-neighbor (NNN) bonds between the planes.

\begin{tabular}{lccc}
\hline \hline$i j$ & type & $w_{i j}^{2}$ & $w_{j i}^{2}$ \\
\hline 34 & $\mathrm{NN}$ & 0.741 & 0.414 \\
$36_{\text {II }}$ & $\mathrm{NN}$ & 0.985 & 0.601 \\
$34_{\text {III }}$ & $\mathrm{NNN}$ & 0.005 & 0.789 \\
36 & $\mathrm{NNN}$ & 1.068 & 0.047 \\
\hline \hline
\end{tabular}

tion to $\Delta P$ (parallel yo $z$ ) can be estimated as $-4 \mu \mathrm{C} / \mathrm{m}^{2}$, which is small due to the strong cancelation between two inequivalent types of bonds. The contribution of next-nearest neighbors is $-134 \mu \mathrm{C} / \mathrm{m}^{2}$, which is consistent with the value $-120 \mu \mathrm{C} / \mathrm{m}^{2}$, obtained in the HF calculations without additional approximations. $\underline{23}$ Thus, the value of $\Delta P$ is mainly determined by the Wannier transfer between next-nearest neighbors in adjacent plane. This is consistent with other magnetic properties of hexagonal manganites. For instance, the type of the magnetic coupling between the planes is also controlled by the NNN interactions, while the contribution of the nearest neighbors is small or can be of the opposite sign. ${ }^{23}$

\section{SUMMARY AND CONCLUSIONS}

In this work, we extend the DE theory of the ME coupling and systematically apply it to the wide class of multiferroic manganites, exhibiting different crystallographic and magnetic structures. For all considered materials, we are able to present a transparent physical picture of how the FE polarization is induced and controlled by the magnetic order. This picture is based on the DE theory, which was formulated for the effective low-energy model, derived 
from the first-principles calculations. Our basic idea is that for the analysis of electronic properties of manganites one can use two physical limits. The first one is the DE limit, which means that the intraatomic exchange splitting between the majority- and minorityspin states, driven by Hund's interactions, is so large that the contribution of the latter states to the electronic polarization can be neglected. The second one is the limit of large intraatomic splitting $\Delta$ between the occupied and unoccupied orbitals with the majority spin, which is driven by the Jahn-Teller distortion and the screened on-site Coulomb repulsion. The second limit allows us to use the perturbation theory for the occupied Wannier functions in the first order of $1 / \Delta$, which can be incorporated in the general Berry-phase theory of polarization. Thus, the electronic polarization can be described in term of the asymmetric transfer of the weights of the Wannier functions to the neighboring sites, which, in the DE model, are additionally modulated by the spin-dependent factors. The DE model allows to greatly simplify the analysis of the polarization and present it in the transparent form, explaining the interplay between the crystallographic and magnetic structures. Moreover, it provides the simple analytical dependence of the FE polarization on the relative directions of spins. As expected for the nonrelativistic theory of the FE polarization, this dependence is given by the 'isotropic correlators' $\mathbf{e}_{i} \cdot \mathbf{e}_{j}$. It has the same form as for the phenomenological magnetostriction mechanism. Nevertheless, this is a new mechanism, which is not directly related to the magnetostriction and can take place without magnetostriction, even in a centrosymmetric crystal structure.

First, we have systematically applied the DE theory to the orthorhombic Pbnm manganites and generalized results of our previous work (Ref. 9). Our present result is valid for any noncollinear magnetic structure, of an arbitrary periodicity, propagating along the orthorhombic $\boldsymbol{b}$ axis and antiferromagnetically coupled along the $\boldsymbol{c}$ axis. For this type of magnetic structures, we have argued that the FE polarization should be parallel to the orthorhombic $\boldsymbol{a}$ axis and can be obtained by scaling the one of the collinear E-type AFM state with the scaling factor depending exclusively on the relative direction of spins. The FE polarization vanishes in the homogeneous spin-spiral state, which preserves the inversion symmetry of the DE Hamiltonian. Therefore, the only possibility to obtain the finite polarization is to deform the homogeneous spin spiral and to produce some inhomogeneity in the distribution of spins. In multiferroic manganites, such a deformation is caused by the relativistic SO interaction. This picture works equally well for manganites with the 
twofold and fourfold periodic magnetic structures, which typically attributed to $\mathrm{HoMnO}_{3}$ and $\mathrm{TbMnO}_{3}$, respectively. The basic difference is that, even despite some spin canting and deviation from the collinear E-type AFM alignment, the twofold periodic magnetic structure remains strongly inhomogeneous, that leads to the large polarization. On the contrary, the fourfold periodic magnetic structure can be viewed as a distorted homogenous spin spiral. Therefore, if the distortion is small, the polarization is also small.

Next, we have studied the microscopic origin of the FE polarization, caused by the magnetic inversion symmetry breaking in the $C 2 / c$ phase of $\mathrm{BiMnO}_{3}$. The uniqueness of this situation is that the magnetic ground state of $\mathrm{BiMnO}_{3}$ contains both $\mathrm{AFM} \uparrow \downarrow \uparrow \downarrow$ component, which breaks the inversion symmetry, and the FM magnetization, caused by the canting of spins. Thus, this is a rare case, where the FE polarization indeed coexists with the FM magnetization, that is very important from the viewpoint of the mutual control of ferroelectricity and magnetism. According to the mean-field HF calculations, the AFM magnetization lies in the monoclinic $z x$ plane, while the FM one is parallel to the $y$ axis. $\frac{22}{2}$ We have modeled this magnetic structure in our DE analysis in order to find a quantitative relationship between the FM magnetization and the FE polarization. As expected, these two quantities 'anticorrelate' with each other: by enforcing the FM magnetization, one can decreases the polarization. The latter can be obtained by scaling the one of the collinear $\uparrow \downarrow \uparrow \downarrow$ AFM state. The scaling factor is given again by the correlation function between directions of neighboring spins, but since the FM moments do not contribute to the polarization, this correlation function includes only AFM components of the magnetization. The polarization in this case lies in the $z x$ plane (so as the AFM magnetization). Moreover, the $x$ component of the polarization is substantially larger than the $z$ one. We have found this behavior to be closely related to the orbital ordering, realized in $\mathrm{BiMnO}_{3}$ : while the $x$ component is very robust and can be expected even in the perfect cubic lattice (provided that it supports the particular type of the orbital ordering, realized in $\mathrm{BiMnO}_{3}$ ), the weak $z$ component is the measure of deviation from the perfect cubic environment, which crucially depends on the details of the monoclinic distortion.

Finally, we have explained the origin of the ME coupling, associated with the reversal of spins in every second $x y$ plane of hexagonal manganites. Such a reversal can be indeed expected in realistic materials if one can induce the change of the magnetic structure from $\Gamma_{2}$ to $\Gamma_{3}$ (or between any two types of the magnetic structures, in which the magnetic moments 
in the neighboring planes are transformed, respectively, by the native symmetry operations of the $P 6_{3} \mathrm{~cm}$ space group and by the same symmetry operations, combined with the time reversal). Although the $P 6_{3} \mathrm{~cm}$ space group has no inversion symmetry and, therefore, the system is expected to be ferroelectric, irrespectively on the magnetic order, this change of the magnetic structure produced a finite change of the FE polarization. We have derived an analytical expression for the spin-dependent part of the polarization and evaluated different contributions to it, associated with the transfer of the weights of the Wannier functions to different groups of sites in the adjacent planes. We have found that the main contribution comes from next-nearest neighbors, while the ones from the nearest sites are small due to the strong cancelation, which occurs between two inequivalent types of bonds.

In conclusion, the DE mechanism of the ME coupling plays a very important role in physics of multiferroic manganites and explains many basics aspects of the FE activity in these systems on a unified ground. Thus, this is the key microscopic mechanisms, which should be considered in the analysis of multiferroic properties of manganites and related compounds.

Acknowledgements. This work is partly supported by the grant of Russian Science Foundation (project No. 14-12-00306).

* SOLOVYEV.Igor@nims.go.jp

1 W. Eerenstein, N. D. Mathur, and J. F. Scott, Nature 442, 760 (2006); T. Kimura, Annu. Rev. Mater. Res. 37, 387 (2007); S.-W. Cheong and M. Mostovoy, Nature Materials 6, 13 (2007); D. Khomskii, Physics 2, 20 (2009); Y. Tokura and S. Seki, Adv. Mater. 22, 1554 (2010).

2 H. Katsura, N. Nagaosa, and A. V. Balatsky, Phys. Rev. Lett. 95, 057205 (2005).

3 I. A. Sergienko, C. Şen, and E. Dagotto, Phys. Rev. Lett. 97, 227204 (2006).

4 S. Picozzi, K. Yamauchi, B. Sanyal, I. A. Sergienko, and E. Dagotto, Phys. Rev. Lett. 99, 227201 (2007).

5 R. V. Aguilar, M. Mostovoy, A. B. Sushkov, C. L. Zhang, Y. J. Choi, S-W. Cheong, and D. H. Drew, Phys. Rev. Lett. 102047203 (2009).

6 M. Mochizuki, N. Furukawa, and N. Nagaosa, Phys. Rev. Lett. 104177206 (2010); ibid. 105 037205 (2010). 
7 I. A. Sergienko and E. Dagotto, Phys. Rev. B 73, 094434 (2006).

8 T. Arima, J. Phys. Soc. Jpn. 76073702 (2007).

9 I. V. Solovyev and S. A. Nikolaev, Phys. Rev. B 87, 144424 (2013).

10 P. Barone, K. Yamauchi, and S. Picozzi, Phys. Rev. Lett. 106, 077201 (2011).

11 K. Yamauchi and P. Barone, J. Phys.: Condens. Matter 26, 103201 (2014).

12 The magnetic structure of many multiferroic manganites is organized in such a way that there is certain group of Mn sites, which are transformed to themselves by the regular inversion. This typically contradicts to the symmetry properties of some other group of Mn atoms, which, due to specific form of interatomic magnetic interactions, should be transformed to themselves by the inversion combined with the time reversal operation. In order to satisfy simultaneously these two symmetry constraints, one of atomic groups should become nonmagnetic. However, it would correspond to a gigantic loss of intraatomic Hund's energy. Therefore, it is more favorable energetically to preserve the high spin state of $\mathrm{Mn}^{3+}$, but to break the inversion symmetry.

13 C. Zener, Phys. Rev. 82440 (1951); P. W. Anderson and H. Hasegawa, Phys. Rev. 100675 (1955); P.-G. de Gennes, Phys. Rev. 118141 (1960); K. Kubo and N. Ohata, J. Phys. Soc. Jpn. 3321 (1972).

14 E. Dagotto, T. Hotta, and A. Moreo, Phys. Rep. 3441 (2001).

15 I. V. Solovyev and K. Terakura, Phys. Rev. Lett. 83, 2825 (1999); I. V. Solovyev, Phys. Rev. B 63, 174406 (2001).

16 T. Hotta, M. Moraghebi, A. Feiguin, A. Moreo, S. Yunoki, and E. Dagotto, Phys. Rev. Lett. 90, 247203 (2003).

17 R. D. King-Smith and D. Vanderbilt, Phys. Rev. B 47, 1651 (1993); D. Vanderbilt and R. D. King-Smith, ibid. 48, 4442 (1993).

18 R. Resta, J. Phys.: Condens. Matter 22, 123201 (2010).

19 I. V. Solovyev, J. Phys.: Condens. Matter 20, 293201 (2008).

20 I. V. Solovyev, Phys. Rev. B 83, 054404 (2011).

21 I. V. Solovyev, M. V. Valentyuk, and V. V. Mazurenko, Phys. Rev. B 86, 144406 (2012).

22 I. V. Solovyev and Z. V. Pchelkina, Pis'ma Zh. Eksp. Teor. Fiz. 89, 701 (2009) [JETP Lett. 89, 597 (2009)]; I. V. Solovyev and Z. V. Pchelkina, Phys. Rev. B 82, 094425 (2010). Note that theoretical values of the FE polarization, reported in the last paper (and also in Ref. 20), were oversetimated by about 2.5 because of numerical error. 
23 I. V. Solovyev, M. V. Valentyuk, and V. V. Mazurenko, Phys. Rev. B 86, 054407 (2012).

24 K. Yamauchi, F. Freimuth, S. Blügel, and S. Picozzi, Phys. Rev. B 78, 014403 (2008).

25 D. Okuyama, S. Ishiwata, Y. Takahashi, K. Yamauchi, S. Picozzi, K. Sugimoto, H. Sakai, M. Takata, R. Shimano, Y. Taguchi, T. Arima, and Y. Tokura, Phys. Rev. B 84, 054440 (2011).

26 W. Kohn and L. J. Sham, Phys. Rev. 140, A1133 (1965); R. G. Parr and W. Yang, Densityfunctional theory of atoms and molecules (Clarendon Press, Oxford, 1989); W. Kohn, Rev. Mod. Phys. 71, 1253 (1999).

27 I. Solovyev, J. Phys. Soc. Jpn. 78, 054710 (2009).

28 N. Marzari, A. A. Mostofi, J. R. Yates, I. Souza, and D. Vanderbilt, Rev. Mod. Phys. 84, 1419 (2012).

29 F. Aryasetiawan, M. Imada, A. Georges, G. Kotliar, S Biermann, and A. I. Lichtenstein, Phys. Rev. B 70, 195104 (2004).

30 O. K. Andersen, Phys. Rev. B 12, 3060 (1975); O. Gunnarsson, O. Jepsen, and O. K. Andersen, ibid. 27, 7144 (1983); O. K. Andersen, Z. Pawlowska, and O. Jepsen, ibid. 34, 5253 (1986).

31 T. Moriya, J. Appl. Phys. 391042 (1968).

32 T. Kimura, T. Goto, H. Shintani, K. Ishizaka, T. Arima, and Y. Tokura, Nature 426, 55 (2003).

33 S. Ishiwata, Y. Kaneko, Y. Tokunaga, Y. Taguchi, T. Arima, and Y. Tokura, Phys. Rev. B 81, $100411(2010)$.

34 A. Muñoz, M. T. Casáis, J. A. Alonso, M. J. Martínez-Lope, J. L. Martínez, and M. T. Fernández-Díaz, Inorg. Chem. 40, 1020 (2001).

35 T. Kimura, G. Lawes, T. Goto, Y. Tokura, and A. P. Ramirez, Phys. Rev. B 71, 224425 (2005).

36 T. Arima, T. Goto, Y. Yamasaki, S. Miyasaka, K. Ishii, M. Tsubota, T. Inami, Y. Murakami, and Y. Tokura, Phys. Rev. B 72, 100102 (2005).

37 K. I. Kugel and D. I. Khomskii, Sov. Phys. Usp. 25, 231 (1982).

38 R. Seshadri and N. A. Hill, Chem. Mater. 13, 2892 (2001).

39 A. A. Belik, S. Iikubo, T. Yokosawa, K. Kodama, M. Igawa, S. Shamoto, M. Azuma, M. Takano, K. Kimoto, Y. Matsui, and E. Takayama-Muromachi, J. Am. Chem. Soc. 129, 971 (2007); A. A. Belik, J. Solid State Chem. 195, 32 (2012).

40 P. Baettig, R. Seshadri, and N. A. Spaldin, J. Am. Chem. Soc. 129, 9854 (2007).

41 I. V. Solovyev, Phys. Rev. B 90, 024417 (2014).

42 Supplemental materials [model parameters for $\mathrm{BiMnO}_{3}$ and hexagonal $\mathrm{YMnO}_{3}$ ]. 
43 J. C. Slater and G. F. Koster, Phys. Rev. 94, 1498 (1954).

44 S. Lee, A. Pirogov, M. Kang, K.-H. Jang, M. Yonemura, T. Kamiyama, S.-W. Cheong, F. Gozzo, N. Shin, H. Kimura, Y. Noda, and J.-G. Park, Nature 451, 805 (2008).

45 A. Muñoz, J. A. Alonso, M. J. Martínez-Lope, M. T. Casáis, J. L. Martínez, and M. T. Fernández-Díaz, Phys. Rev. B 62, 9498 (2000).

46 P. J. Brown and T. Chatterji, J. Phys.: Condens. Matter 18, 10085 (2006). 